\title{
A STUDY OF THE COLLISIONAL EVOLUTION OF ORBITAL DEBRIS IN GEOPOTENTIAL WELLS AND GEO DISPOSAL ORBITS
}

\author{
A Thesis \\ presented to \\ the Faculty of California Polytechnic State University \\ San Luis Obispo
}

\author{
In Partial Fulfillment \\ of the Requirements for the Degree \\ Master of Science in Aerospace Engineering
}

by

Christina Diaz

July 2013 
(C) 2013

Christina Diaz

ALL RIGHTS RESERVED 


\section{COMMITTEE MEMBERSHIP}

TITLE:

A Study of the Collisional Evolution of Orbital Debris in Geopotential Wells and GEO Disposal Orbits

AUTHOR: $\quad$ Christina Diaz

DATE SUBMITTED: July 2013

COMMITTEE CHAIR: Kira J. Abercromby, Ph.D, Aerospace Engineering, Professor

COMmitTeE MEMBER: Gary Hughes, Ph.D, Statistics, Professor

COMMITTEE MEMBER: Paula Krisko, Ph.D, NASA Johnson Space Center, Orbital Debris Modeling Specialist

COMMITTEE MEMBER: Jordi Puig-Suari, Ph.D, Aerospace Engineering, Professor 


\begin{abstract}
A Study of the Collisional Evolution of Orbital Debris in Geopotential Wells and GEO Disposal Orbits

Christina Diaz

This thesis will present the effects of the orbital debris evolution in two key areas: the geosynchronous disposal orbit regime known as "graveyard" and the two geopotential wells found in $105^{\circ} \mathrm{W}$ and $75^{\circ} \mathrm{E}$ longitude bins. After developing a GEO specific orbit propagator for NASA Johnson Space Center's Orbital Debris Office, collisions were simulated throughout these regimes using a low velocity breakup model. This model considered the effects of perturbations particularly non-spherical Earth effects (specifically sectorial and zonal harmonics), lunar effects, third body effects and solar radiation pressure effects. The results show that CDPROP does well in simulating the presence of the Eastern and Western geopotential wells, as well as catching drifting GEO objects. It does not do as well in catching East-West trapped objects. Three collision test cases were then simulated in graveyard and the East and West geopotential wells.
\end{abstract}




\section{Acknowledgments}

It has been a long road and I am glad to have, in the smallest way, expanded on the emerging field of understanding GEO debris. I would like express my sincere gratitude to NASA Johnson Space Center's Dr. Paula Krisko and Dr. Phillip AnzMeador at the Orbital Debris Office for their extensive assistance and dedication in this thesis with the Fortran programming language and understanding modeling orbital debris. They offered me great direction and insight. It has been a wonderful learning experience and opportunity for me.

I would also like to give a great thanks to Dr. Kira Abercromby for her motivating words and assistance in this thesis as my committee chair, professor, and mentor. Her belief and enthusiasm in me was one of my driving forces in completing this

thesis. I would also like to thank her for helping me discover the interesting fields of astrodynamics and space environments through her classes and research.

A big thank you as well to Dr. Jordi Puig-Sari and Dr. Gary Hughes who also helped throughout my time working on my thesis with whatever support they could give.

And as always, I would like to thank my loved ones for their unconditional support and understanding throughout my time working to complete this thesis. I can now leave my cave, formally known as my computer desk.

-Christina 


\section{Contents}

List of Tables $\quad$ viii

List of Figures $\quad$ ix

1 Introduction and Background 1

1.1 GEO and Near GEO Region . . . . . . . . . . . . . . . 2

1.1.1 Geosynchronous Orbit . . . . . . . . . . . . . 2

1.1 .2 GEO Graveyard . . . . . . . . . . . . . . . . . 4

1.2 About the Two Line Element Set . . . . . . . . . . . . 6

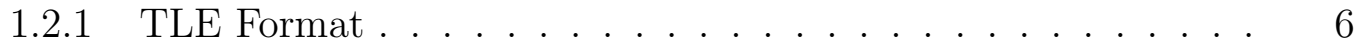

1.3 Current Work . . . . . . . . . . . . . . . . . . . . 8

1.3.1 Early Work . . . . . . . . . . . . . . . . 8

1.3.2 Revisiting GEO and Near-GEO . . . . . . . . . . . 8

1.3.3 Reassessing GEO Disposal and Geopotential Well Risk . . . . 9

1.4 Thesis Novelty . . . . . . . . . . . . . . . . . . . 10

2 Cataloged GEO Debris 12

3 Orbital Debris Collisions 16

3.1 Hypervelocity vs. Low Velocity Break Ups . . . . . . . . . . 17

4 Methodology 19

4.1 Propagation . . . . . . . . . . . . . . . . . . . . 19

4.1.1 Perturbation Analysis . . . . . . . . . . . . . . . 19

4.1.2 Choosing a Propagation Method . . . . . . . . . . . . . 23

4.2 The Breakup Model . . . . . . . . . . . . . . . . . . . . . . . . . 24

4.2 .1 Size Distribution . . . . . . . . . . . . . 25 
$4.2 .2 \quad \mathrm{~A} / \mathrm{m}$ Distribution . . . . . . . . . . . . . 26

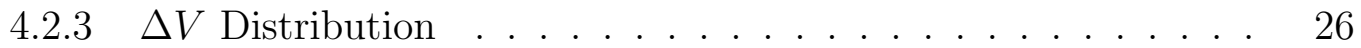

5 CDPROP - Fortran 90 Propagator 27

5.1 Functionality . . . . . . . . . . . . . . . . . . . . 28

5.1 .1 Test File . . . . . . . . . . . . . . . . . . . . . . 30

5.1 .2 User Input . . . . . . . . . . . . . . . . . . . . . . 31

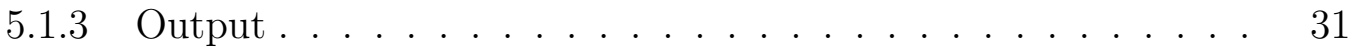

5.2 Verification and Validation . . . . . . . . . . . . . . . 32

5.2 .1 Verification Using Titan . . . . . . . . . . . . . . . . . 32

5.2.2 Validation using Selected TLEs . . . . . . . . . . . . . 35

5.2 .3 Residuals from Historical Data . . . . . . . . . . . . . . . 42

5.2 .4 Conclusion . . . . . . . . . . . . . . . . . . . . . 43

6 Application of CDPROP: Collision Modeling 45

6.1 The Example Cases . . . . . . . . . . . . . . . . . . . . 45

6.2 Results . . . . . . . . . . . . . . . . . . 46

6.2.1 Graveyard Collision Results _. . . . . . . . . . . . . . . 48

6.2 .2 East Well Collision Results . . . . . . . . . . . . . . . . 50

6.2 .3 West Well Collision Results . . . . . . . . . . . . . . . 52

7 Conclusion and Future Work $\quad 58$

$\begin{array}{ll}\text { Bibliography } & 60\end{array}$ 


\section{List of Tables}

1.1 These elements are contained in the first line of the TLE. . . . . . . 7

1.2 These elements are contained in the second line of the TLE. . . . . . 7 


\section{List of Figures}

1.1 Locations of the geopotential wells are $75^{\circ} \mathrm{E}$ and $105^{\circ} \mathrm{W}$ longitude bins [1]. . . . . . . . . . . . . . . . . . . . . .

1.2 It can be shown based on longitude bin and $\Delta \Delta$ which well a satellite will drift towards [1]

2.1 Some current graveyard and GEO Debris used were found using available NORAD SatCat TLE data. . . . . . . . . . . . . . . . . . . . .

2.2 Latitude distribution of objects along GEO arc is shown. Note most

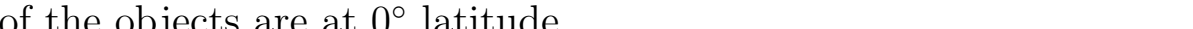

2.3 Current debris objects trapped in geopotential wells with available ESA DISCOS data is shown. Red represents the Eastern Well $\left(75^{\circ} \mathrm{E}\right)$, blue represents the Western Well $\left(105^{\circ} \mathrm{W}\right)$, and black represents objects in libration between the East and West Wells. . . . . . . . . . . . . . .

2.4 Latitude Distribution of objects trapped or a in libration orbit between GPW is shown. Notice the longitudes where clumping occurs affirms the presence of a GPW. Red represents the Eastern Well $\left(75^{\circ} \mathrm{E}\right)$, blue represents the Western Well $\left(115^{\circ} \mathrm{W}\right)$, and black represents objects in libration between the East and West Wells. .....

2.5 The pink highlighted area reflects the $105^{\circ} \mathrm{W}$ geopotential well and the blue-green highlighted area reflects the $75^{\circ} \mathrm{E}$ geopotential well.

4.1 The direction of $\vec{r}_{\oplus \odot}$ is crucial for the sign convention of Equation 4.7

5.1 A general flowdown of CDPROP shows the key components of the program - reading in user input, performing Cowell's method, and then writing to a text file. . . . . . . . . . . . . . . . . .

5.2 Terminal output on the version of gcc fortran used. More specific information can be found on http://gcc.gnu.org . . . . . . . 29

5.3 Example input during an execution of CDPROP is shown. . . . . . . 29 
5.4 An example of how user should compile and execute the testfile to check compiler was installed properly. . . . . . . . . . . . . .

5.5 An overview of the orbital path of STK's simulation and CDPROP's simulation. STK is in blue while CDPROP is in magenta. . . . . . 33

5.6 Position residuals over 10 days are shown. . . . . . . . . . . . 34

5.7 Velocity residuals over 10 days are shown. . . . . . . . . . . . 34

5.8 Comparison of four objects' orbits over a few periods. The red trajectory is Skynet 1. The blue trajectory is OPS 9432. The black trajectory is Fengyun 2D and the green is ATS $5 \ldots \ldots \ldots \ldots \ldots$

5.9 Comparison of eccentricity between objects shows Fengyun has the highest eccentricity while ATS5 has the lowest. . . . . . . . . . 36

5.10 Comparison of inclination between objects shows similar behavior for Skynet, OPS, and ATS 5; however, Fengyun changes inclination quite differently. . . . . . . . . . . . . . . . . . . 37

5.11 Longitudinal history of ATS 5 depicts characteristic drifter behavior. 38

5.12 Longitudinal history of Fengyun 2D depicts characteristic trapped behavior, in this case the Eastern geopotential well. . . . . . . . . . .

5.13 Longitudinal history of Skynet 1 depicts characteristic trapped behavior, in this case the Western geopotential well. . . . . . . . . . . .

5.14 Longitudinal history of OPS 9432 does not show the characteristic behavior of EW trapped objects. . . . . . . . . . . . . . . 41

5.15 The OPS 9432 satellite according to ESA's DISCOS database should be bound from $190^{\circ}$ to $540^{\circ}$, right in between the East and West wells. 41

5.16 Comparison of ATS 5's historical data and CDPROP's simulation. . .

5.17 A generation of the first derivative of mean motion values showing suspected values. . . . . . . . . . . . . . . .

6.1 $\Delta \mathrm{V}$ distribution shows the majority of fragments ejected are between

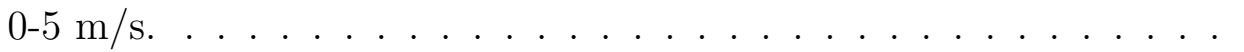

6.2 This figure depicts the general idea behind finding the fragments new state vectors for propagation. $\Delta \mathrm{V}$ is a product of the distribution magnitude and direction unit vectors. This is then added to the current velocity and the position vector is assumed to be the same, instantaneously. . . . . . . . . . . . . . . . . .

6.3 A theoretical graveyard collision was simulated in graveyard orbit in this location. 
6.4 A theoretical graveyard collision was simulated in graveyard orbit. The magenta orbit represents the slowest imparting debris fragment while the cyan orbit represents the fastest imparting debris fragment. Note the Earth is not necessarily positioned with geographical accuracy. . .

6.5 A theoretical collision was simulated in an Eastern well. The location is shown. Note the Earth is not necessarily positioned with geographical accuracy. . . . . . . . . . . . . . . . . .

6.6 A theoretical collision was simulated in an Eastern well. The magenta orbit represents the slowest imparting debris fragment while the cyan orbit represents the fastest imparting debris fragment. Note the Earth is not necessarily positioned with geographical accuracy. . . . . . .

6.7 The longitudinal history of the collision's slowest and fastest fragments is shown. It initially displays characteristic behavior of a East-West trapped object but eventually loses that behavior in the simulation. .

6.8 A theoretical collision was simulated in a Western well. The location is shown. Note the Earth is not necessarily positioned with geographical accuracy. . . . . . . . . . . . . . . . . . .

6.9 A theoretical collision was simulated in a Western well. The magenta orbit represents the slowest imparting debris fragment while the cyan orbit represents the fastest imparting debris fragment. Note the Earth is not necessarily positioned with geographical accuracy. . . . . . . .

6.10 The longitudinal history of the collision's slowest and fastest fragments is shown. The slowest fragment shows trapped behavior, while the fastest object does not. . . . . . . . . . . . . . . . . . . 55

6.11 The $\Delta \Delta$ values of the East collision fragments are shown. . . . . . . . 56

6.12 The $\Delta \Delta$ values of the West collision fragments are shown. . . . . . . 56 


\section{Chapter 1}

\section{Introduction and Background}

In terms of the space environment, orbital debris is a present and future hazard to all Earth space operations. Orbital debris consists of in-operational man-made objects currently in Earth orbit such as derelict spacecraft, rocket bodies, explosion fragments and even flecks of paint. Debris-generating events such as collisions also contribute to the present population. Orbital debris' time in orbit is dependent on the orbital altitude. Debris in lower orbits often reenter within several years to decades; however, debris above $1000 \mathrm{~km}$ can remain in orbit for a century or more.

With the growing number of satellites being launched into space and no active debris measures currently being taken, understanding debris evolution is critical to the future development of satellite systems. Currently, there are approximately 385 geosynchronous artificial satellites in orbit, and the number grows every year. Geosynchronous is a high traffic, highly-valued real estate due to its orbital characteristics. Communications and Global Position System satellites (GPS) satellites often utilize this region. Currently, mitigation practices suggest that spacecraft must be moved at least $300 \mathrm{~km}$ up at the end of life into a disposal orbit known as graveyard orbit. The spacecraft in this region are nonoperational and are moved here to lower 
the collisional probability with operational spacecraft in the geosynchronous region. Doing this maneuver requires extra fuel at end of life (EOL) as well as an attitude control system to perform the transfer. While most operators try to perform this maneuver, it hard to say that the maneuver is always completed to standard. Also, near this region exist "sinks," caused by a nonspherical Earth gravity field, where debris and other objects will remain "trapped" indefinitely. These two areas are cause for concern because of the high traffic near these regions and the relatively unknown behavior of these trapped objects.

\subsection{GEO and Near GEO Region}

Understanding debris evolution requires a thorough understanding of the orbital characteristics and dynamic environment of higher altitude orbits. This section will discuss the orbital characteristics of those regions.

\subsubsection{Geosynchronous Orbit}

By far one of the most important and essential orbits, the geosynchronous orbit has become the realm of the modern communications and weather-tracking satellite. Geosynchronous spacecraft was a concept developed by the Arthur C. Clark in which he theorized that communications around the world would someday be feasible using a triangulating communication constellation spaced around the equator equally [2]. In 1963, NASA tested Clark's theory through the Synchronous Communications Satellite program. After an electronic failure in Syncom 1, Syncom 2 because the first operational geosynchronous communications satellite, launching on July 23, 1963 [cite]. On August 19, 1964, Syncom 3 because the first geostationary satellite.

A geosynchronous orbit (GSO) is an orbit around the Earth with a orbital pe- 
riod approximately equal to one sidereal day, or 23 hours 56 minutes and 4 seconds, matching the Earth's rotation. This allows for the object in geosynchronous orbit to be in exact same position in the sky after one period. A special space of geosynchronous orbit is the geostationary orbit (GEO) which has a circular orbit with zero inclination, or directly above the equator. Communication satellites often utilize this orbit to accommodate their antenna design and have it permanently in a fixed location. All Earth geosynchronous orbits have a semi-major axis of $42140 \mathrm{~km}$ and thus can be expressed as,

$$
a=\sqrt[3]{\mu\left(\frac{P}{2 \pi}\right)}
$$

where a is the semi-major axis (in $k m$ ), $\mathrm{P}$ is orbital period (in $s$ ), and $\mu$ is Earth's gravitational constant (in $\mathrm{km}^{3} / \mathrm{s}^{2}$ ).

\section{Geopotential Wells}

Because of the effects of a non-spherical Earth, gravitational forces naturally pull objects towards two known "geopotential wells." They are located at the $75^{\circ} \mathrm{E}$ and $105^{\circ} \mathrm{W}$ longitude bins as shown in Figure 1.1. Operational satellites, who require stabilization, use east-west stationkeeping to combat these forces.

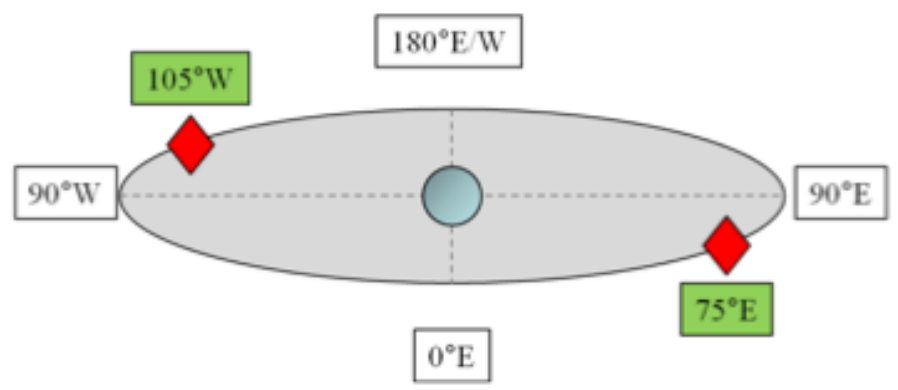

Figure 1.1: Locations of the geopotential wells are $75^{\circ} \mathrm{E}$ and $105^{\circ} \mathrm{W}$ longitude bins [1]. 
Once these satellites become derelict they naturally go to the nearest well and oscillate about its center continuously. There are also objects that are known to drift between the wells in a ping-pong type manner instead of being trapped in a specific well. In regards to orbital debris, geopotential wells are of interest due to their interesting behavior and relatively unknown effect on the debris population. It is assumed that these wells behave like debris "sinks", trapping debris pieces and not releasing them, but it is still relatively unstudied.

\subsubsection{GEO Graveyard}

Until the publication of NASA Safety Standard 1740.14, Guidelines and Assessment Procedures for Limiting Orbital Debris, satellite EOL and disposal options did not have much importance in the design of most spacecraft [3]. EOL practices place a design cost on spacecraft architectures' $\Delta \mathrm{V}$ budgets and mission operations. But with the growing debris problem, particularly in the active LEO and GEO regions, it has become increasingly important for consideration. NASA's standard is not regulated internationally; however, it is strongly suggested [4].

The general options for satellite programs are either (a) direct retrieval and deorbit, (b) maneuver to an orbit for which atmospheric drag will remove the remaining structure within 25 years, or (c) maneuver to a nearby disposal region. Most GEO spacecraft will transfer to the super-GEO storage orbit, also known as graveyard, whereas highly eccentric, high perigee altitude spacecraft might transfer to a subGEO storage orbit rather than lowering perigee to reenter. The Inter-Agency Space Debris Coordination Committee (IADC) dictates that the minimum perigee altitude, $\Delta H$ (in $\mathrm{km}$ ), for a disposal location for an object above a $200 \mathrm{~km}$ altitude in its final mission orbit is

$$
\Delta H=235+\left(1000 C_{R} \frac{A}{m}\right) k m
$$


where $C_{r}$ is the coefficient of solar radiation pressure and $A / m$ is the area to mass ratio of the spacecraft. Either the aforementioned option or maneuvering to a storage orbit between LEO and GEO where the perigee altitude is above $2500 \mathrm{~km}$ and apogee altitude is below $35,288 \mathrm{~km}$ (500 km below GEO altitude) must be completed successfully. A program also must use the post-mission disposal strategy that has the least risk of leaving the vehicle near GEO in the event of a failure during the disposal process. Because of fuel-gauging uncertainties near the end of mission, it is suggested that the maneuver be performed in a series of at least four burns which alternately raise apogee and then perigee. The four burns, prescribed in Guideline 6-2a of the NASA Technical Standard 8719.14, Process for Limiting Orbital Debris, take into account fuel gauging limitations which are particularly serious at the end of all missions [3]. Given that there will be significant uncertainty as to the amount of fuel remaining at the EOL, there will also be some uncertainty as to whether there is enough fuel to complete the disposal maneuvers to standard. If there is not enough fuel to complete the maneuvers, a plan using four smaller burns to maneuver to the disposal orbit will leave the spacecraft or upper stage farther from GEO altitude than would a plan using two burns.

According to the NASA standard, the region of space above GEO altitude can be used as a disposal region with little concern for debris buildup because of the low relative velocities, large regions of available space, and relatively low traffic rates in this area. In the near future, the $300 \mathrm{~km}$ altitude separation will be sufficient to isolate the disposal region from GEO if steps are taken to remove on-board energy sources after completion of the post-mission disposal maneuver. However, depending on the level of traffic to GEO and on the characteristic sizes of future GEO satellites, this separation distance may need to be increased in the future. If measures are not taken to prevent explosive structural failure after disposal of GEO systems, a separation distance of $2000 \mathrm{~km}$ will be required to isolate the disposal region from GEO. 
The purpose of these disposal orbits, otherwise known as "graveyard" is to lower the probability of collisions with operational spacecraft and generate more debris. Disposal orbits are usually used when the $\Delta v$ in order to deorbit the spacecraft is too high. To deorbit a geostationary spacecraft the $\Delta v$ needed is approximately 1.5 $\mathrm{km} / \mathrm{s}$ as opposed to reorbiting it into a graveyard orbit at $0.011 \mathrm{~km} / \mathrm{s}$. This transfer is approximately how much a satellite would need for 3 months of stationkeeping. At this point, in order to perform the maneuver correctly, the satellite must have a reliable attitude control method to complete the transfer.

Having said that, EOL protocol may need to be looked at in the future to make sure the assumptions made today are valid years from now.

\subsection{About the Two Line Element Set}

A two-line element set (TLE) is a commonly used data format used to explain sets of orbital elements that describe the position of a specific object or satellite at a given time. The format is specified by the North American Aerospace Command (NORAD). NORAD maintains general perturbation element sets on all resident space objects. The objects are periodically updated. It is important to note that the elements are mean values and are obtained by removing periodic variations in the orbital elements.

\subsubsection{TLE Format}

The formatting of the TLEs is as follows,

AAAAAAAAAAAAAAAAAAAAAAAA

1 NNNNNU NNNNNAAA NNNNN. NNNNNNNN +. NNNNNNNN +NNNNN-N +NNNNN-N N NNNNN

2 NNNNN NNN. NNNN NNN. NNNN NNNNNNN NNN. NNNN NNN. NNNN NN. NNNNNNNNNNNNNNN 
where A represents a character and $\mathrm{N}$ represents a number. The first line is a twenty four character satellite name. The next two lines describe the orbital characteristics as described in Tables 1.1 and 1.2 by the column number and their description.

\begin{tabular}{|c|c|}
\hline Column & Description \\
\hline 01 & Line Number of Element Data \\
$03-07$ & Satellite Number \\
08 & Classification (U = Unclassified) \\
$10-11$ & International Designator (Last two digits of launch year) \\
$12-14$ & International Designator (Launch number of the year) \\
$15-17$ & International Designator (Piece of the launch) \\
$19-20$ & Epoch Year (Last two digits of year) \\
$21-32$ & Epoch (Day of the year and fractional portion of the day) \\
$34-43$ & First Time Derivative of the Mean Motion \\
$45-52$ & Second Time Derivative of Mean Motion (decimal point assumed) \\
$54-61$ & BSTAR drag term (decimal point assumed) \\
63 & Ephemeris type \\
$65-68$ & Element number \\
69 & Checksum (Modulo 10) \\
\hline
\end{tabular}

Table 1.1: These elements are contained in the first line of the TLE.

\begin{tabular}{|c|c|}
\hline Column & Description \\
$03-07$ & Line Number of Element Data \\
$09-16$ & Satellite Number \\
$18-25$ & Inclination [Degrees] \\
$27-33$ & Eight Ascension of the Ascending Node [Degrees] \\
$35-42$ & Argument of Perigee [Degrees] \\
$44-51$ & Mean Anomaly [Degrees] \\
$53-63$ & Mean Motion [Revs per day] \\
$64-68$ & Revolution number at epoch [Revs] \\
69 & Checksum (Modulo 10) \\
\hline
\end{tabular}

Table 1.2: These elements are contained in the second line of the TLE.

These lines are then parsed using Fortran 90 and then used appropriately to get the position and velocity vectors of the object. 


\subsection{Current Work}

In the past decade, interest has been placed in characterizing the environment near and in the GEO regime, particularly after satellites have finished their mission and stationkeeping is no longer used. The foundation of this thesis lies within the work to be mentioned below.

\subsubsection{Early Work}

In 1986, Jozef C. Van der Ha of the European Space Operations Center, developed a model for the long-term evolution of free-drifting near-GEO satellites in "Long-Term Evolution of Near-Geostationary Orbits". His evolution model modified the perturbation equations in order to discard short term orbital element variation, particularly orbits with a period of less than 24 hours [5]. Perturbations included in Van der Ha's analysis were lunisolar gravitational forces up to the second parallactic term of moon, zonal and tesseral harmonics of the Earth's potential field up the fourth degree, as well as solar radiation forces. His results have allowed prediction to be made regarding spacecraft at the EOL and without stationkeeping. It was validated using ESA's GOES-2 satellite.

\subsubsection{Revisiting GEO and Near-GEO}

In "A New Look at GEO and Near-GEO Regimes: Operations, Disposals, and Debris", Nicholas J. Johnson stated that the orbital characteristics of objects in and near GEO differed greatly from the classic geostationary case once used before in analysis. First, modern GEO satellites use more complex orbits utilizing inclination

and eccentricity for their specific mission [6]. Also, the natural perturbations such as non-spherical Earth gravity and solar radiation pressure effects have a significant 
effect on the movement of objects. Johnson also looked at several of the surveys done of the GEO region and how observations have revealed a significant population that is small and potentially hazardous. These objects cannot be found in satellite catalogs. Disposal at EOL for GEO spacecraft was recommended to preserve the GEO environment for future missions and operations.

\subsubsection{Reassessing GEO Disposal and Geopotential Well Risk}

In 2011, Darren S. McKnight and Frank R. Di Pentino analyzed orbit collision hazard to operational spacecraft in GEO. The goal was to find a clear linkage between orbital characteristics and resulting risk. From their research, several insights were found. First, the collisional probability in GEO is relatively low; however, because of the lack of understanding of the present environment, the future is very difficult to access [1]. This is mainly due to observational limitation and lack of information regarding historical and future debris-generating events. Second, the probability of collision in GEO is dependent on longitude. It was found to be seven times greater in regions near geopotential wells, specifically the $105^{\circ} \mathrm{W}$ and $75^{\circ} \mathrm{E}$ wells. Third, it was also found through simulation that the probability of critical mission failure was largely dependent on the 2200 objects between $30 \mathrm{~cm}$ to $1 \mathrm{~m}$ currently observed in GEO, but uncatalogued. The fourth insight was that despite previous assessment that "graveyard" disposal orbits were relatively safe to avoid collision risk, they actually pose a potential additional risk that is not yet fully understood. Lastly, collision hazard was seen to be non-uniform throughout the course of the day or year. One of the most interesting things found was the relationship between orbital characteristics and risk of being trapped in a geopotential well. This was known as the $\Delta \Delta$ criteria. $\Delta \Delta$ is a function of the perigee (smallest radius of orbit) and apogee (largest radius of orbit) of the object relative to the GEO arc when "decommissioned". The relation 
is shown to be,

$$
\Delta \Delta=\left(42164-r_{a}\right)-\left(42164-r_{p}\right)
$$

where $r_{a}$ is radius at apogee (in $\mathrm{km}$ ), $r_{p}$ is radius at perigee (in $\mathrm{km}$ ). According to previous work, empirical and analytical investigations have shown that any derelict object with a $\Delta \Delta \leq 40 \mathrm{~km}$ will be trapped no matter where along the GEO arc it is abandoned. However, longitude has an effect on the $\Delta \Delta$ value. The closer to the geopotential well the longitude is the $\Delta \Delta$ may range up to $80 \mathrm{~km}$ and still get trapped. Another exception is that the object does not need to be close to a well in order to get trapped. An example is Cosmos 1586 payload, which had a perigee 93 $\mathrm{km}$ above the GEO arc and an apogee $101 \mathrm{~km}$ above the GEO arc. It had a $\Delta \Delta$ of $8 \mathrm{~km}$ and is currently stuck in the Eastern well.

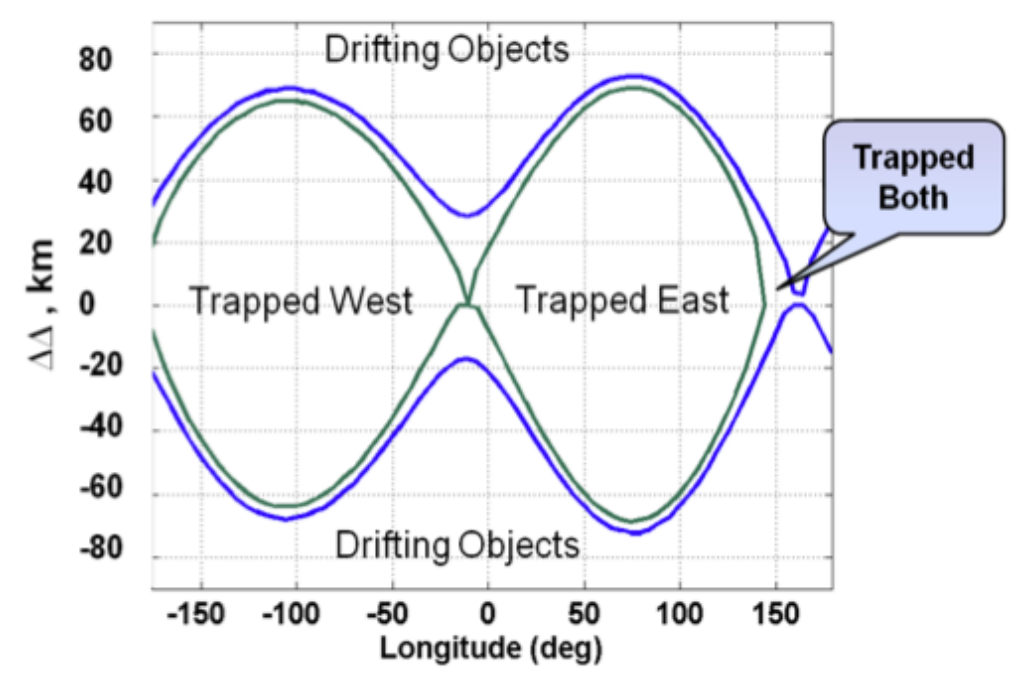

Figure 1.2: It can be shown based on longitude bin and $\Delta \Delta$ which well a satellite will drift towards [1].

\subsection{Thesis Novelty}

The novelty of this thesis lies in the relative uncertainty known about the GEO and near-GEO region and its implication on the future of the environment. One 
of the main reasons for this uncertainty is the observation limitations. In order to discover an object's trajectory, several observations are taken and then used to get a two line element or TLE of the object, which tells the orbital characteristic of the object. In GEO, observatories, such as the MODEST Observatory, can currently see objects as small as $30 \mathrm{~cm}$; however, tracking it is where the limitations come into play [7]. Assuming this is the current boundary on observations, it is easy to see how the GEO debris population is underestimated. In low Earth orbit (LEO), fragments smaller than $10 \mathrm{~cm}$ are known to exist, the same is plausible in GEO. The population of smaller fragments need to be considered in future environmental studies as well as considered in future spacecraft risk mitigation and post-operational life.

Along with observational limitations, it is difficult to access the historical and future of debris-generating events without understanding breakups in this region. Unlike in LEO where collisions are known to be hypervelocity impacts (i.e. $6 \mathrm{~km} / \mathrm{s}$ ), GEO collisions happen much slower, along the realm of $1-3 \mathrm{~km} / \mathrm{s}$. This brings the need for the development and validation of a high accuracy low velocity breakup model. Currently, there is work being done experimentally to base a model after the NASA Standard Breakup Model, currently used in lower orbits, but until then, the behavior of impacts will remain uncertain.

With that being said, without understanding the graveyard environment fully, debris from a future collision may cause similar problems like that seen in lower orbits. The issue could be even more catastrophic due to the high traffic volume associated to the GEO and near GEO regions. This thesis seeks to study specific problems to help further the understanding of debris evolution in this region. 


\section{Chapter 2}

\section{Cataloged GEO Debris}

In order to begin studying the GEO environment, a database of currently tracked objects needed to be collected. It is important to note that amount of debris objects tracked and available is very limited. Debris objects in graveyard and geopotential wells were collected from two sources, NORAD's SatCat and ESA's DISCOS database. These objects are shown in Figures 2.1, 2.2, 2.3, and 2.4.

Graveyard and GEO objects were collected from the NORAD SatCat database. Satellites (mainly payloads) that had periods greater than 1445 minutes were chosen. Geosynchronous orbits had a period of 1436 minutes. The objects were then requesting through a special data request on the NORAD SatCat database. A total of 202 objects were found.

Objects specifically in the geopotential wells were collected from the European

Space Agency's DISCOS database based on the following parameters: (a) Eccentricity must be smaller than 0.2. (b) Mean motion must be between 0.9 and 1.1 revolutions per sidereal day,corresponding approximately to a semi-major axis between 42164$2500 \mathrm{~km}$ and $42164+3150 \mathrm{~km}$. (c) Inclination must be lower than $30^{\circ}$. The orbital characteristics do not come with designated uncertainty values, but this was taken 
into account in the analysis.

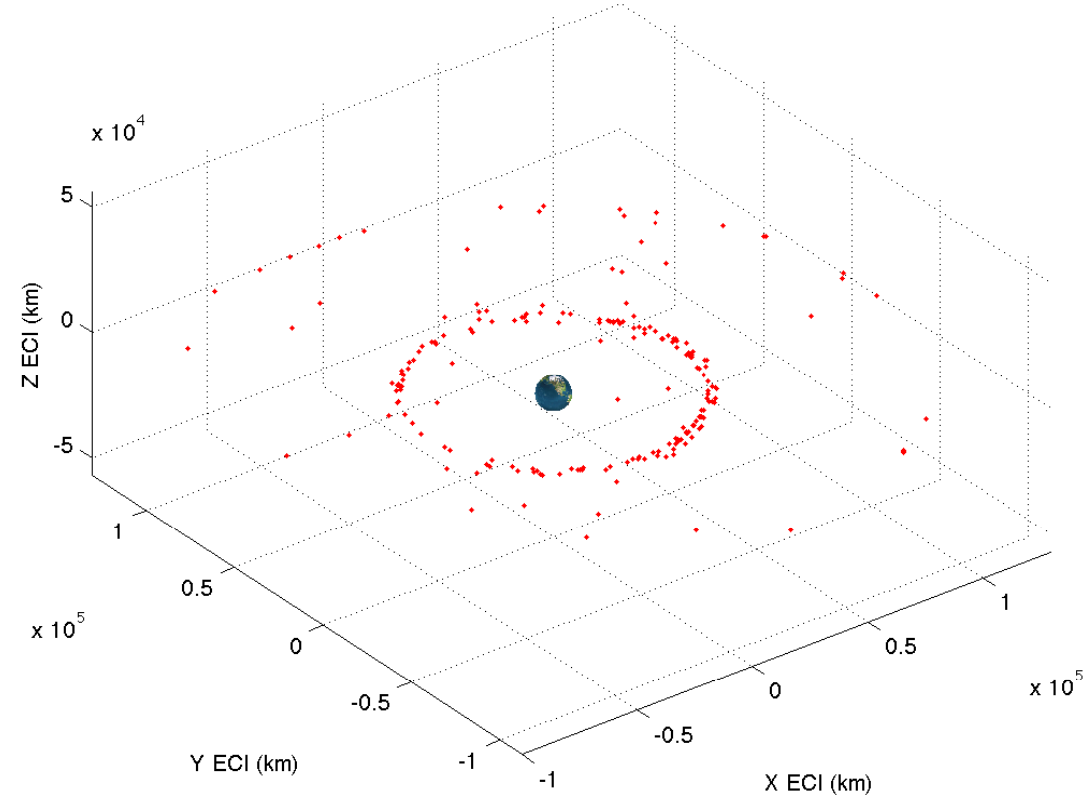

Figure 2.1: Some current graveyard and GEO Debris used were found using available NORAD SatCat TLE data.

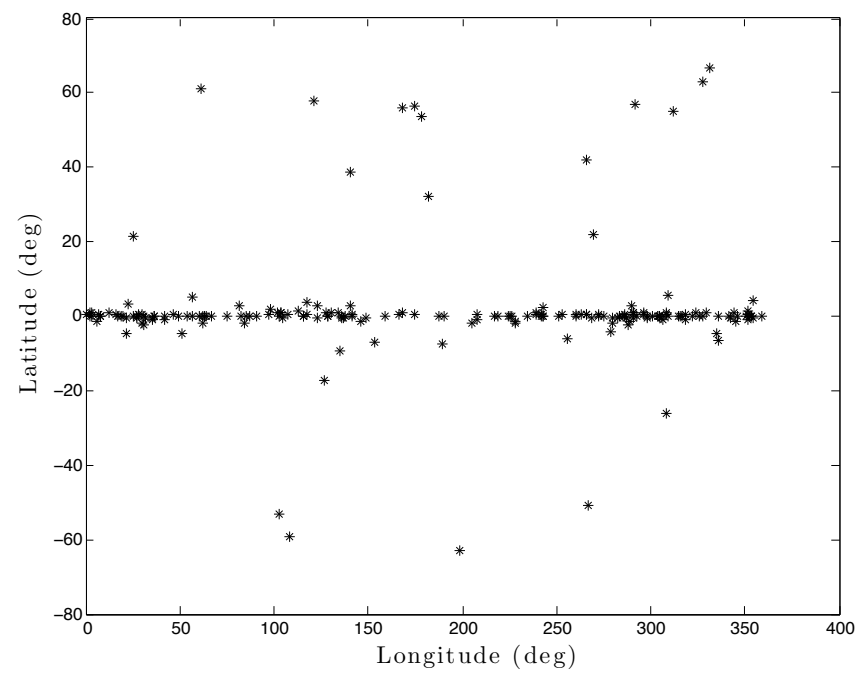

Figure 2.2: Latitude distribution of objects along GEO arc is shown. Note most of the objects are at $0^{\circ}$ latitude

Figure 2.2 shows the somewhat uniform distribution of GEO objects in the GEO 


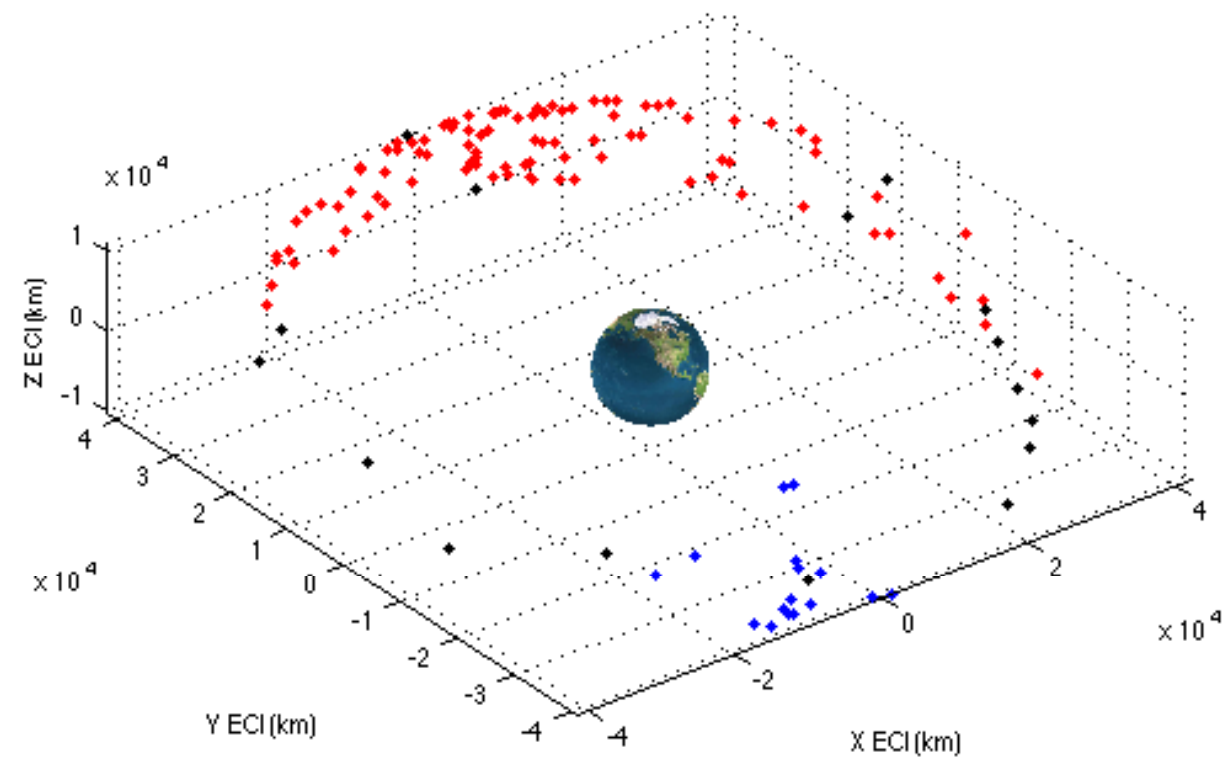

Figure 2.3: Current debris objects trapped in geopotential wells with available ESA DISCOS data is shown. Red represents the Eastern Well $\left(75^{\circ} \mathrm{E}\right)$, blue represents the Western Well $\left(105^{\circ} \mathrm{W}\right)$, and black represents objects in libration between the East and West Wells.

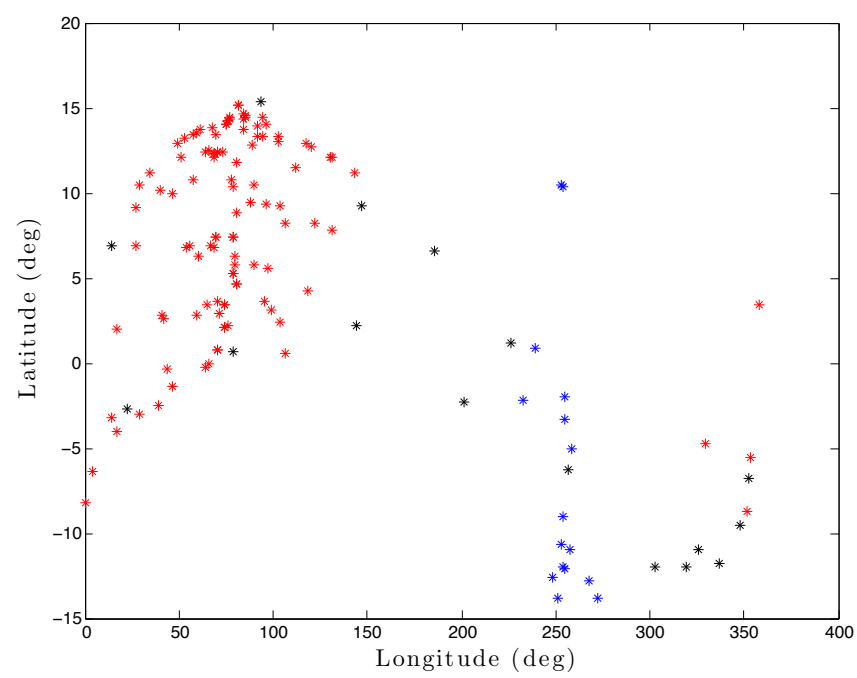

Figure 2.4: Latitude Distribution of objects trapped or a in libration orbit between GPW is shown. Notice the longitudes where clumping occurs affirms the presence of a GPW. Red represents the Eastern Well $\left(75^{\circ} \mathrm{E}\right)$, blue represents the Western Well $\left(115^{\circ} \mathrm{W}\right)$, and black represents objects in libration between the East and West Wells. 
arc. It verifies that most satellites can be found within $+/-15^{\circ}$. The oscillation in inclination is caused by the combination of lunar and solar body gravity effects. Figure 2.4 shows the characteristic clumping of objects that designate the presence of a geopotential well. It is interesting to note that the reasoning for more objects being found found in the Eastern well is not due to a "stronger" well, but rather lack of strict EOL practices. The location of the wells relative on the map are shown in Figure 4.1.

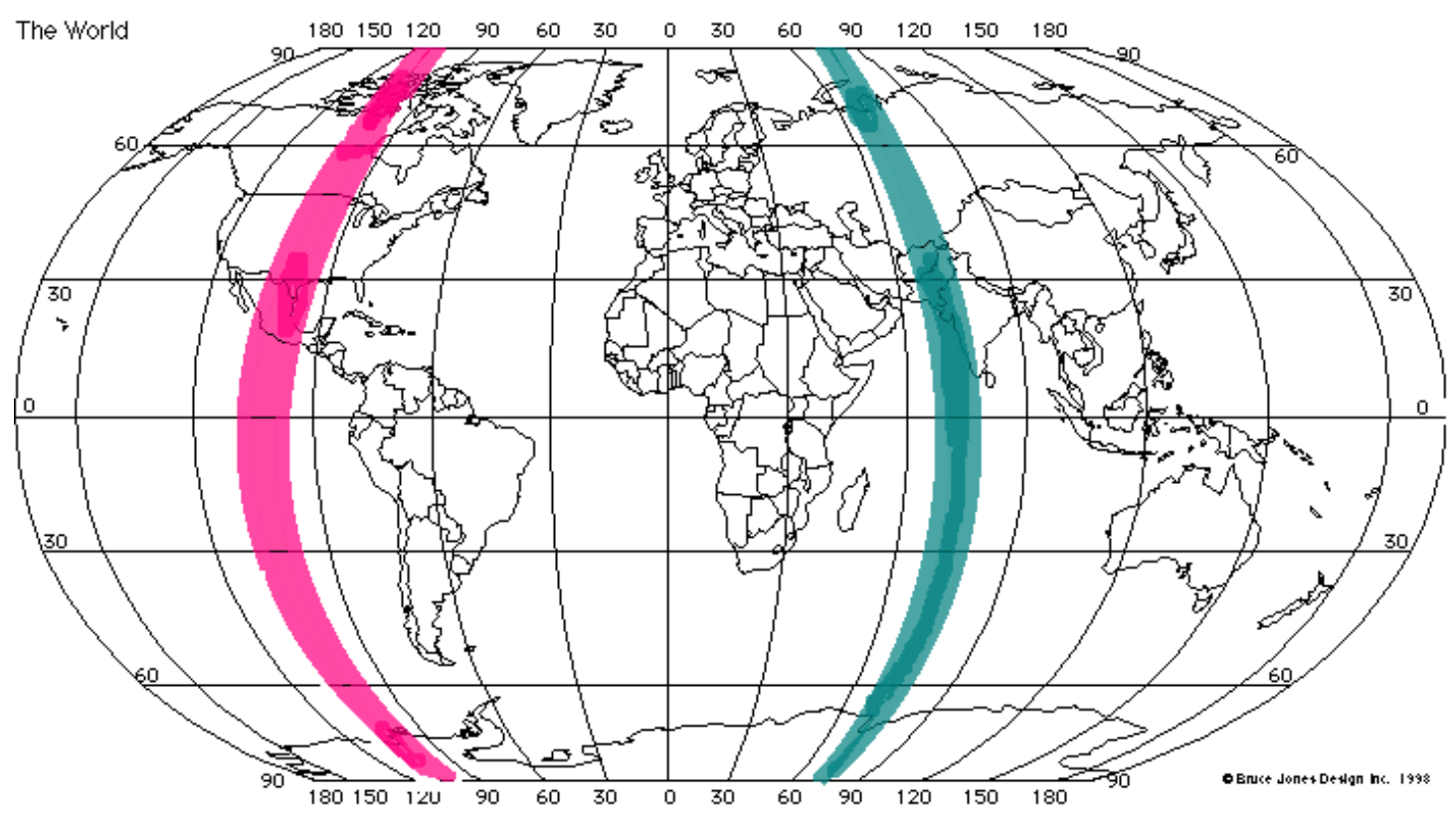

Figure 2.5: The pink highlighted area reflects the $105^{\circ} \mathrm{W}$ geopotential well and the blue-green highlighted area reflects the $75^{\circ} \mathrm{E}$ geopotential well. 


\section{Chapter 3}

\section{Orbital Debris Collisions}

In 1978, Donald Kessler and Burton Cour-Palais published their work, "Collision Frequency of Artificial Satellites: The Creation of a Debris Belt." It was theorized that as the number of artificial satellites in Earth orbit increases, collision probability between satellites also increases. These collisions would produce more orbiting fragments which again increase the probability of future collisions. This cyclic process would eventually lead to the development of a debris belt around the Earth. They stated that this belt could begin to form as early as within the century and would continue to cause problems in the future to spacecraft design and mission operations. With their findings, it was emphasized that more research would need to take place to understand debris collisions as well as develop a better model to make future assessments on alleviating the issue [8]. The direness of debris collisions projected in the future could result in a debris ring around the Earth analogous to the ringed systems Saturn and Uranus due to the Earth's Roche's limit at $9000 \mathrm{~km}$ [9]. From this, it can be seen how important it is to accurately assess the space environment by accounting for future collisions as well as accurately model them. 


\subsection{Hypervelocity vs. Low Velocity Break Ups}

Up until recently, the majority of work regarding orbital debris collisions had been focused on LEO fragmentation. Objects in LEO orbit have speeds from 6-7 $\mathrm{km} / \mathrm{s}$. Collision velocities at this range are considered to be hypervelocity. In order to understand collisions, a model must be created to define the parameters before and after the collision. A satellite breakup model, at a minimum, should define the size, area-to-mass ratio, and ejection velocity of each generated fragment.Since these parameters are not constant for all debris, distributions as a function of a given parameter, e.g., mass or characteristic length, are necessary. In addition, the initial conditions of the breakup, e.g., the total mass of the parent object or the collision velocity, can be highly influential [10]. It is important to also include or address variances in the derived distributions since multiple break events of the same type of objects will not produce the same debris cloud each time [10]. Most models are derived from Earth based hypervelocity impact tests and observed data from on-orbit collisions. The simulated debris clouds are more accurately represented by the fragments seen in orbit and in laboratory. This mainly because the previous models did correctly assess debris. First, it treated all fragmentation debris as spheres of a density which varied as a function of fragment diameter, where diameter was directly related to mass. Second, it underestimated the generation of fragments smaller than 10-cm in the majority of explosions [10]. From this it can be seen finding an accurate breakup model is indeed very difficult. The larger caveat is that current debris collision models only apply to LEO orbits, since they are validated with LEO type velocities.

For studying the GEO and near-GEO region, a hypervelocity model cannot be used. This is mainly due to the fact that collisions occur at much slower speeds. The orbital velocity itself is in the realm of $1-3 \mathrm{~km} / \mathrm{s}$. Thus, the relative velocity 
between most collisions is approximately $800 \mathrm{~m} / \mathrm{s}$. In order to accurately access the environment a more realistic model must be applied. Work is currently being done to find a low velocity model. The most developed one found was based off the NASA Breakup Model itself and scaled down based on a series of low velocity impact ground-based tests. Work was done at the NASA Johnson Space Center by Toshi Hanada in attempt to scale down the NASA Breakup Model to be applied to a GEO collision. Using the NASA Standard Breakup Model 1998 revision, Hanada's experiment showed agreement. His findings are summarized below [11].

1. The size distribution model adequately assesses fragment trends, but should be modified to not underestimate the number of fragments greater than a given size.

2. The size-to-area conversion matches low velocity experimental data.

3. The area-to-mass $(\mathrm{A} / \mathrm{M})$ distribution model did not show good agreement, but it was thought that it could still be applied to a low velocity collision because this difference could be a cause of differences in impact material used in tests and not impact velocities.

4. NASA $\Delta V$ distribution model does not show good agreement for a low-velocity collision so it had to be scaled.

With that said, Hanada's model will be used to analyze debris clouds during simulated collisions. The model will be presented in detail in Ch 4 . 


\section{Chapter 4}

\section{Methodology}

\subsection{Propagation}

To adequately access the evolution orbital debris over time, a perturbation analysis must be done. In order to accurately analyze the effect of the Earth's natural disturbance on debris, a numerical analysis method was needed for orbit propagation. Besides considering computational efficiency, choice of propagation method was dependent on the types of perturbing forces considered- nonconservative, conservative, or both.

\subsubsection{Perturbation Analysis}

In the GEO and near-GEO regime, the most significant perturbations are caused by solar radiation pressure, nonspherical Earth gravity, solar and lunar body gravity effects. With that being said, these were the perturbing accelerations that were considered in the orbit propagator developed for this thesis, Collisional Debris Propagator or CDPROP for short. It will be described in much greater detail in Chapter 5 , for now, the thought process to its development is presented. 


\section{Nonspherical Earth}

Because the gradient of the potential for a spherical central body will yield acceleration on an object, it must be examined how the potential will be altered due to a nonspherical central body. The following expression for the gravitational has been adopted by the 1961 International Astronomical Union [12]. This expression describes the gravitational attraction resulting from the irregular distribution of the Earth's mass using a potential function and is expressed as,

$$
U=\frac{\mu_{\oplus}}{r}\left[1+\sum_{l=2}^{\infty} \sum_{m=0}^{\ell}\left(\frac{R_{\oplus}}{r}\right)^{\ell} P_{\ell, m}\left[\sin \left(\phi_{g c}\right)\right]\left\{C_{\ell, m} \cos \left(m \lambda_{s a t}\right)+S_{l, m} \sin \left(m \lambda_{s a t}\right)\right\}\right]
$$

where $U$ is the potential function, $\mu_{\oplus}$ is the gravitational parameter of the Earth (in $\mathrm{km} / \mathrm{s}^{2}$ ), $r$ is the magnitude of the orbiting object's position vector (in $\mathrm{km} / \mathrm{s}$ ), $R_{\oplus}$ is the radius of the Earth in (in $\mathrm{km}$ ), $\phi_{g c}$ is the geocentric latitude (in radians), $\lambda_{\text {sat }}$ is the satellite's geocentric longitude (in radians), $\ell$ and $m$ represents the gravitational model's order [12]. In Eqn. (4.1), $P_{\ell, m}$ is represented the Legendre polynomial and $C_{\ell, m}$ and $S_{\ell, m}$ are the gravitational coefficients found using the EGM-96 model. This model was chosen due to its high accuracy derived from numerous satellite observations and measurements. It has since replaced the JGM-2 model [12]. From Eqn.(4.1), the derivatives with respect to $r, \phi_{g c}$ and $\lambda_{s a t}$. These are shown as

$$
\begin{gathered}
\frac{\partial U}{\partial r}=-\frac{\mu_{\oplus}}{r^{2}} \sum_{l=2}^{\infty} \sum_{m=0}^{\ell}\left(\frac{R_{\oplus}}{r}\right)^{\ell}(\ell+1) P_{\ell, m}\left[\sin \left(\phi_{g c}\right)\right] \\
\times\left\{C_{\ell, m} \cos \left(m \lambda_{s a t}\right)+S_{l, m} \sin \left(m \lambda_{s a t}\right)\right\} \\
\frac{\partial U}{\partial \phi_{g c}}=\frac{\mu_{\oplus}}{r^{2}} \sum_{l=2}^{\infty} \sum_{m=0}^{\ell}\left(\frac{R_{\oplus}}{r}\right)^{\ell}\left(P_{\ell, m+1}\left[\sin \left(\phi_{g c}\right)\right]-m \tan \left(\phi_{g c}\right) P_{\ell, m}\left[\sin \left(\phi_{g c}\right)\right]\right) \\
\times\left\{C_{\ell, m} \cos \left(m \lambda_{s a t}\right)+S_{l, m} \sin \left(m \lambda_{s a t}\right)\right\} \\
\frac{\partial U}{\partial \lambda_{s a t}}=\frac{\mu_{\oplus}}{r^{2}} \sum_{l=2}^{\infty} \sum_{m=0}^{\ell}\left(\frac{R_{\oplus}}{r}\right)^{\ell} m P_{\ell, m}\left[\sin \left(\phi_{g c}\right)\right] \\
\times\left\{S_{l, m} \cos \left(m \lambda_{s a t}\right)-C_{\ell, m} \sin \left(m \lambda_{s a t}\right)\right\}
\end{gathered}
$$


From the derivatives the acceleration caused by the nonspherical Earth gravity field can be calculated using the following expressions,

$$
\begin{aligned}
& a_{I}=\left\{\frac{1}{r} \frac{\partial U}{\partial r}-\frac{r_{K}}{r^{2} \sqrt{r_{I}^{2}+r_{J}^{2}}} \frac{\partial U}{\partial \phi_{g c}}\right\} r_{I}-\left\{\frac{1}{r_{I}^{2}+r_{J}^{2}} \frac{\partial U}{\partial \lambda_{s a t}}\right\} r_{J} \\
& a_{J}=\left\{\frac{1}{r} \frac{\partial U}{\partial r}-\frac{r_{K}}{r^{2} \sqrt{r_{I}^{2}+r_{J}^{2}}} \frac{\partial U}{\partial \phi_{g c}}\right\} r_{J}+\left\{\frac{1}{r_{I}^{2}+r_{J}^{2}} \frac{\partial U}{\partial \lambda_{s a t}}\right\} r_{I} \\
& a_{K}=\frac{1}{r} \frac{\partial U}{\partial r} r_{K}+\frac{\sqrt{r_{I}^{2}+r_{J}^{2}}}{r^{2}} \frac{\partial U}{\partial \phi_{g c}} .
\end{aligned}
$$

These accelerations (in $\mathrm{km} / \mathrm{s}^{2}$ ) were then added to the original two body unperturbed acceleration to account for the nonspherical Earth gravity perturbation.

\section{Solar Radiation Pressure}

Solar radiation pressure is a nonconservative force. At higher orbital altitudes, its consideration becomes increasing important. The general case includes several assumption and as each assumption is removed an additional layer of complexity is added in. The main generalizations is that the Sun's flux is a constant $1367 \mathrm{~W} / \mathrm{m}^{2}$, area facing the sun is the same as the velocity direction, there are only three possible values for coefficient of reflectivity, $C_{r}$, and the object is always in sunlight. The force due to solar radiation pressure is expressed as

$$
\vec{F}_{S R P}=-P_{S R P} C_{R} A_{\odot} \vec{r}_{\oplus \odot}
$$

where $\vec{F}_{S R P}$ is solar radiation pressure force (in $N$ ), $\mathrm{P}$ is solar radiation pressure (in $N / m^{2}$ ), and $\vec{r}_{\oplus \odot}$ is the position vector from the object to the sun (in $\mathrm{km}$ ). The value for $C_{r}$ can range from 0 to 2, depending on the object. Materials that are translucent to incident radiation are rated 0 , black body objects are rated 1 , and entirely reflective materials are rated 2. From this, the perturbing acceleration due to solar radiation 
pressure is found using

$$
\vec{a}_{S R P}=\frac{-P_{S R P} C_{R} A \odot}{m} \frac{\vec{r}_{\oplus \odot}}{\left\|\vec{r}_{\oplus \odot}\right\|}
$$

where $\vec{a}_{S R P}\left(\right.$ in $k m / s^{2}$ ) is the perturbing acceleration due to solar radiation pressure $\mathrm{m}$ is the satellite mass (in $\mathrm{kg}$ ). $\vec{r}_{\oplus \odot}$ is best described in the diagram below.

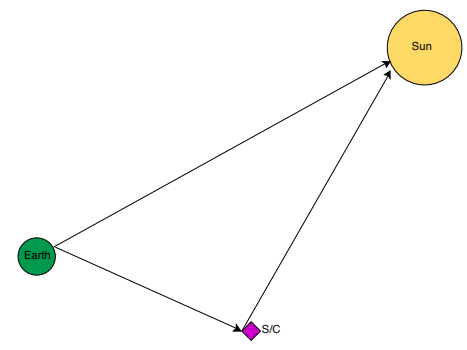

Figure 4.1: The direction of $\vec{r}_{\oplus \odot}$ is crucial for the sign convention of Equation 4.7

This is then added to the running sum of perturbing accelerations, $\vec{a}_{\text {perturbed }}$.

\section{Lunar and Solar Body Effects}

In order to calculate the disturbance of the lunar and solar bodies on the object, the following expression was used based on Newton's Law of Gravitation:

$$
\vec{a}_{b o d y}=G \sum_{j=3}^{n} m_{j}\left(\frac{\vec{r}_{s / c}}{r_{s / c}^{3}}-\frac{\vec{r}_{1 j}}{r_{1 j}^{3}}\right)
$$

where $\vec{a}_{b o d y}$ is the perturbing acceleration due to the lunar and solar body disturbance (in $\left.\mathrm{km} / \mathrm{s}^{2}\right), G$ is the gravitational constant (in $\left.N(\mathrm{~m} / \mathrm{kg})^{2}\right), m$ is the mass (in $\mathrm{kg}$ ) of the $j^{\text {th }}$ body, $\vec{r}_{1 j}$ is the position vector from the central body to the $j_{t h}$ body. This expression works for $n$ bodies besides the central body and orbiting object. For the purposes of CDPROP, only the Sun and moon's body perturbation was considered. 


\subsubsection{Choosing a Propagation Method}

The three main methods of orbit propagation are Cowell's method, Encke's formulation, and Variation of Parameters. When choosing a propagation method, it is important to note the advantages and caveats with each methodology. A trade study was conducted in order to see which method would best suit the needs of this program.

Cowell's Method: the main advantage is that this propagation method is very easy to implement. It's process is through direct integration and is a good method to use when the perturbation is on the same order as the primary acceleration. Smaller perturbation accelerations can cause for instabilities due to truncation error. Computationally speaking, this method requires more integration steps, which in turn means more computational power.

Encke's Formulation: This method is not commonly used today because computers can easily perform the numerical solution with enough precision to retain the accuracy need [12]. It is important to note as its concept can easily be integrated to other methods. Encke's method which begins with the osculating orbital elements (as opposed to the mean elements). Instead of integrating all the forces on the body, Encke only looks at the different between the two body acceleration and the perturbed acceleration. The process continues until the osculating orbit gets too far away. This point is called the rectification point, where the osculating orbit is reinitialized by setting it equal to the two body acceleration. Encke's requires less integration than Cowell's since it only integrates the perturbation and allows large step sizes (which is great for interplanetary trajectories); however, it's implementation is more complex.

Variation of Parameters (VoP): Variation of Parameters is a methodology that works well for small forces and focuses on how the classical orbital elements change with time. Although, VoP is a great methodology, it is particularly expensive, 
difficult to implement, and breaks down for near-circular orbits, which essentially ruled this Gaussian method out.

Method Chosen: Looking at the aforementioned, Cowell's formulation was chosen as the propagation method due to needing to look at both conservative and nonconservative perturbations as well as not wanting any limitations to orbit types. To implement Cowell's formulation, the following equation was used:

$$
\ddot{\vec{r}}=-\frac{\mu}{r^{3}} \vec{r}+\vec{a}_{\text {perturbed }}
$$

where $\vec{a}_{\text {perturbed }}$ is the total acceleration (in $\mathrm{km} / \mathrm{s}^{2}$ ) caused by other forces acting on the object, such as non-spherical central body, atmospheric drag, third-body effects, solar-radiation pressure, and tides, which were discussed in Section 4.1.1.

\subsection{The Breakup Model}

Commonly when studying collisions, a breakup model is applied. These models are usually developed through experimentation and on-orbit collision observations. The currently known NASA Standard Breakup Model as aforementioned is only validated for LEO collisions; however, Hanada's experimental work has allowed this model to be scaled for lower velocity collisions in and near the GEO regime [11]. For any type of collisions, impact velocity is defined by the difference of velocity vectors of the two colliding objects which is expressed as,

$$
V^{2}=V_{1}^{2}+V_{2}^{2}-V_{1} V_{2} \cos \theta
$$

where $V_{1}$ and $V_{2}$ are the pre-collision velocities magnitudes of object 1 and 2 (in $\mathrm{km} / \mathrm{s}$ ), and $\theta$ is the angle between the two velocity vectors (in radians). GEO 
objects are initially in the equatorial plane and change in inclination due to natural perturbations such as lunar and solar body gravitational perturbations and Earth's oblateness. The change in inclination begins with a rate of 0.8 deg per year but then shifts its direction after reaching a maximum of $15^{\circ}$ by 27 years [11]. This affects how collisions are distributed compared to LEO. If an object under north-south station keeping is hit by another object, the angle of collision must be less than $15^{\circ}$. All orbits are essentially circular at the nominal geostationary altitude and objects in inclined orbits make daily appearances in N-S direction relative to stationary satellites. The collision velocity in GEO tend to be less than $800 \mathrm{~m} / \mathrm{s}$, since the orbital velocity in GEO is about $3 \mathrm{~km} / \mathrm{s}$. If the collision is with a GEO satellite and GTO object, the collision velocity increases to $1.5 \mathrm{~km} / \mathrm{s}$ which ends up corresponding to the required velocity to insert a satellite into GEO from GTO.

\subsubsection{Size Distribution}

The size distribution was scaled to give,

$$
N\left(L_{c}\right)=0.6\left(m_{c}\right)^{0.75}\left(L_{c}\right)^{-1.71}
$$

where $L_{c}$ is characteristic length of the fragment (in $m$ ), $N\left(L_{c}\right)$ is the size distribution of $L_{c}$, and $m_{c}$ is the mass (in $\mathrm{kg}$ ) of both objects in the case of a catastrophic collision [11]. If the collision were non-catastrophic, the value of $\mathrm{m}$ is defined as the product of the mass of the smaller object and the square of the collision velocity (in $\mathrm{km} / \mathrm{s}$ ), giving the ejecta mass (in $\mathrm{kg}$ ). The smaller object is assumed to be destroyed in every collision, and its mass is added to the ejecta mass. 


\subsection{2 $A / m$ Distribution}

The area to mass ratio, $A / m$, distribution was scaled as,

$$
D_{A / m}^{S O C}\left(\lambda_{c}, \chi\right)=N\left(\chi ; \mu^{S O C}\left(\lambda_{c}\right), \sigma^{S O C}\left(\lambda_{c}\right)\right)
$$

where $\mu^{S O C}$ is given by,

$$
\mu^{S O C}\left(\lambda_{c}\right)= \begin{cases}-0.3 & \lambda_{c} \leq-1.75 \\ -0.3-1.4\left(\lambda_{c}+1.75\right) & -1.75<\lambda_{c}<-1.25 \\ 1.0 & \lambda_{c} \geq-1.25\end{cases}
$$

and $\sigma^{S O C}$ is,

$$
\sigma^{S O C}\left(\lambda_{c}\right)= \begin{cases}0.2 & \lambda_{c} \leq-3.5 \\ 0.2+0.1333\left(\lambda_{c}+3.5\right) & \lambda_{c}>-3.5\end{cases}
$$

where $\lambda_{c}$ is $\log _{10}\left(L_{c}\right), \chi$ is $\log _{10}(A / M), \nu$ is the $\log _{10}(\Delta V), \sigma$ is the standard deviation, and $\mu$ is the mean [11].

\subsection{3 $\Delta V$ Distribution}

In order to find the normal distribution of $\Delta V, A / M$ was used as the independent variable instead of $L_{c}$. Using the same relationship as the NASA Standard Breakup Model, the following was used,

$$
\begin{gathered}
D_{\Delta V}^{C O L L}(\chi, \nu)=N\left(\nu ; \mu^{C O L L}(\chi), \sigma^{C 0 L L}(\chi)\right) \\
\mu^{C O L L}(\chi)=0.45 \chi+1.45 \\
\sigma^{C O L L}(\chi)=0.27
\end{gathered}
$$

where $\chi$ is $\log _{10}(A / M), \sigma$ is the standard deviation, $\nu$ is the $\log _{10}(\Delta V)$. where $\lambda_{c}$ is $\log _{10}\left(L_{c}\right), \chi$ is $\log _{10}(A / M), \nu$ is the $\log _{10}(\Delta V), \sigma$ is the standard deviation, and $\mu$ is the mean [11]. 


\section{Chapter 5}

\section{CDPROP - Fortran 90 Propagator}

As mentioned previously, the development of a GEO-specific orbit propagator was needed in order to study the debris environment. Using the methodology described in Ch. 4.1, CDPROP, or Collisional Debris Propagator was developed. CDPROP's main objective was to be able to accurately propagate a GEO object with regards to the most specific perturbations to the region - solar radiation pressure, nonspherical Earth effects, and lunar/solar body effects. The orbital information would be parsed from a user-specified TLE text file and other satellite inputs. The text file should include satellite name and then the elemental data in the following lines. Then, CDPROP takes the given parameters and performs Cowell's method using a RungeKutta Felhberg integration method. Once the integration reaches the final time, the resulting state vectors and classical orbital elements are written to a text file along with the respective time stamp. Figure 5.1 describes the process in a bit more detail. The following chapter will give more detailed information on CDPROP's user parameters as well as the program's verification and validation. 


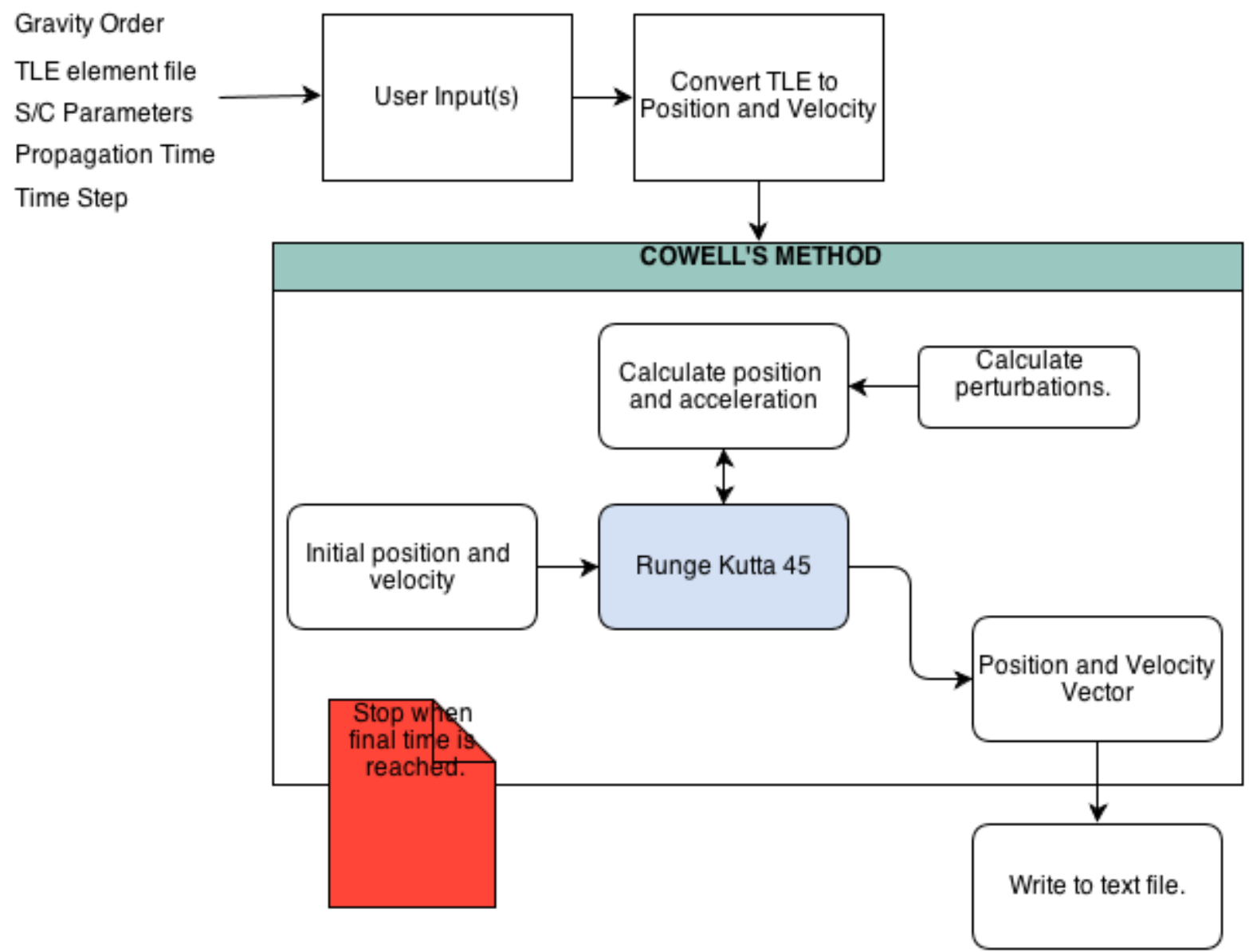

Figure 5.1: A general flowdown of CDPROP shows the key components of the program - reading in user input, performing Cowell's method, and then writing to a text file.

\subsection{Functionality}

CDPROP is a Fortran 90 based computer program. It was tested using a gcc gfortran compiler on Mac OS X. This compiler was chosen mainly for being open source and readily available. The GNU Fortran compiler supports Fortran77, 90, and 95 standards as well as some Fortran 2003 and 2008 standards. Source files with .f, .for, .fpp, .ftn, .F, .FOR, .FPP, and .FTN extensions are treated as fixed form. Source files with .f90, .f95, .f03, .f08, .F90, .F95, .F03 and .F08 extensions are treated as free form. The capitalized versions of either form are run through preprocessing. In order to execute CDPROP, the following method was 


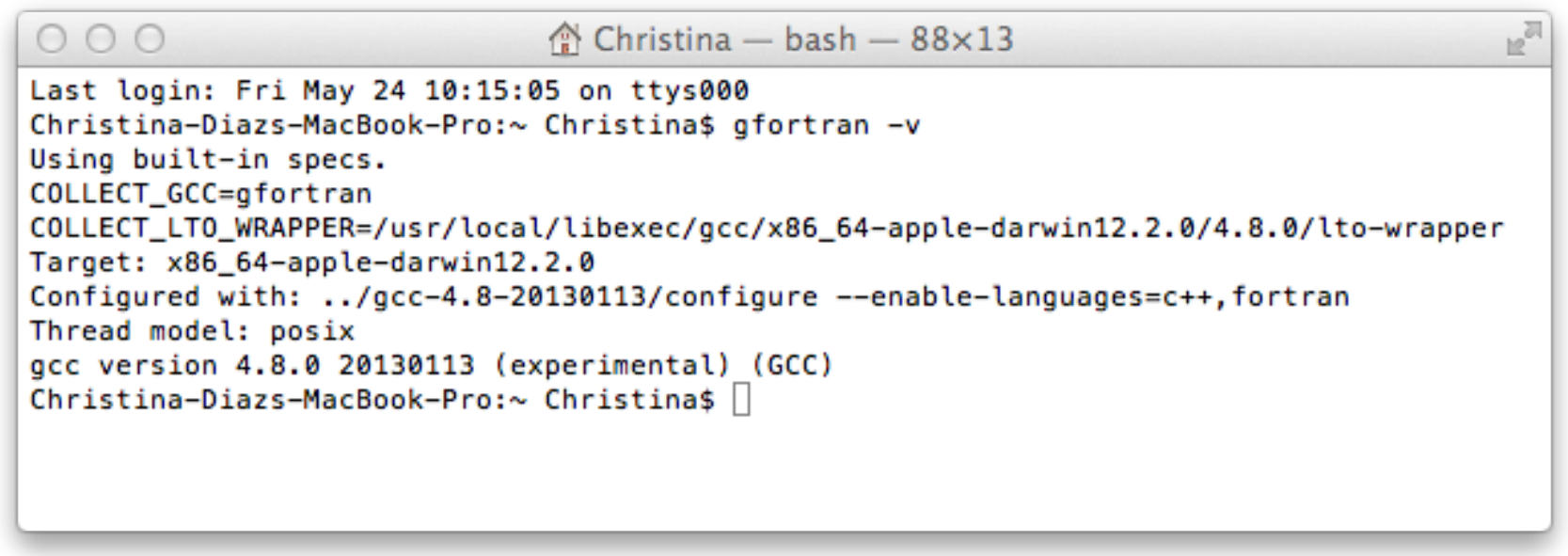

Figure 5.2: Terminal output on the version of gcc fortran used. More specific information can be found on http://gcc.gnu.org

used:

Last login: Fri May 24 10:41:44 on ttys001
Christina-Diazs-MacBook-Pro: Christina\$ cd Dropbox/Thesis/CDPROP
Christina-Diazs-MacBook-Pro:CDPROP Christina\$ gfortran CDPROP. f90
Christina-Diazs-MacBook-Pro:CDPROP Christina\$ ./a.out
Welcome to CDPROP - GE0 Orbit Propagator
Written by: Christina Diaz, 2013
For: NASA JSC Orbital Debris Office
Object Currently Selected:
Titan
Please input the following parameters
Propagation time (days):

Figure 5.3: Example input during an execution of CDPROP is shown. 


\subsubsection{Test File}

In order to ensure the compiler was set up properly as well as the input parameters of CDPROP were entered correctly, a test file was created, testfile.f90. Users should compiler and execute that file exactly as shown below. The user designated inputs are boxed in purple in Figure 5.4.

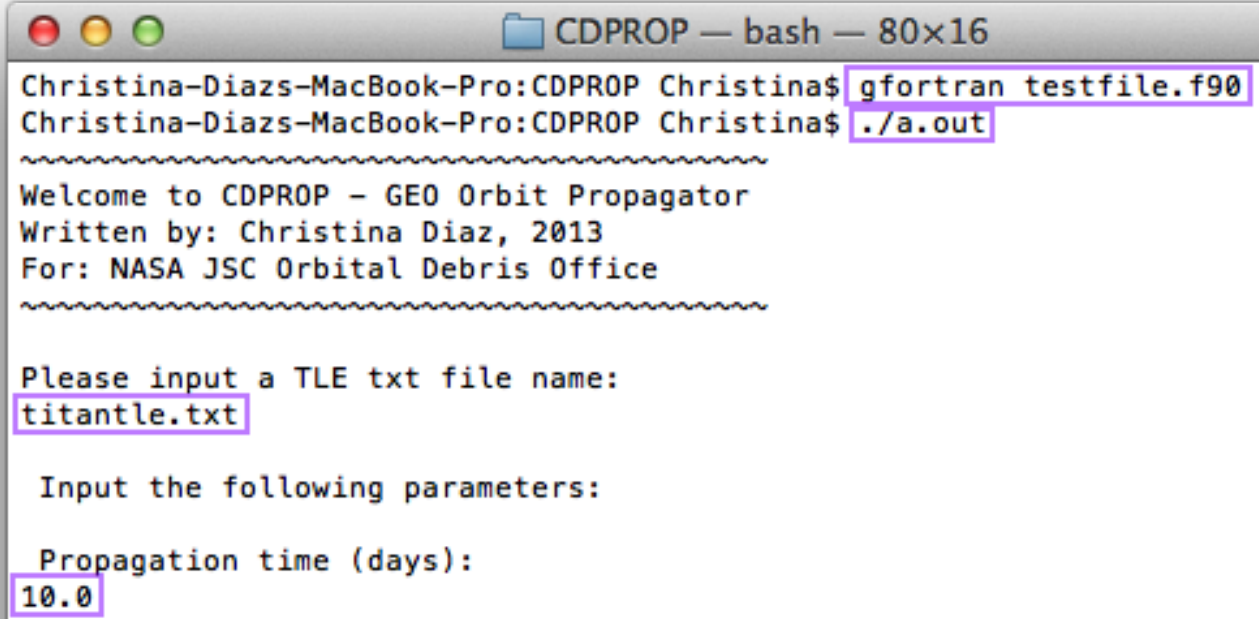

Figure 5.4: An example of how user should compile and execute the testfile to check compiler was installed properly.

The results will be:

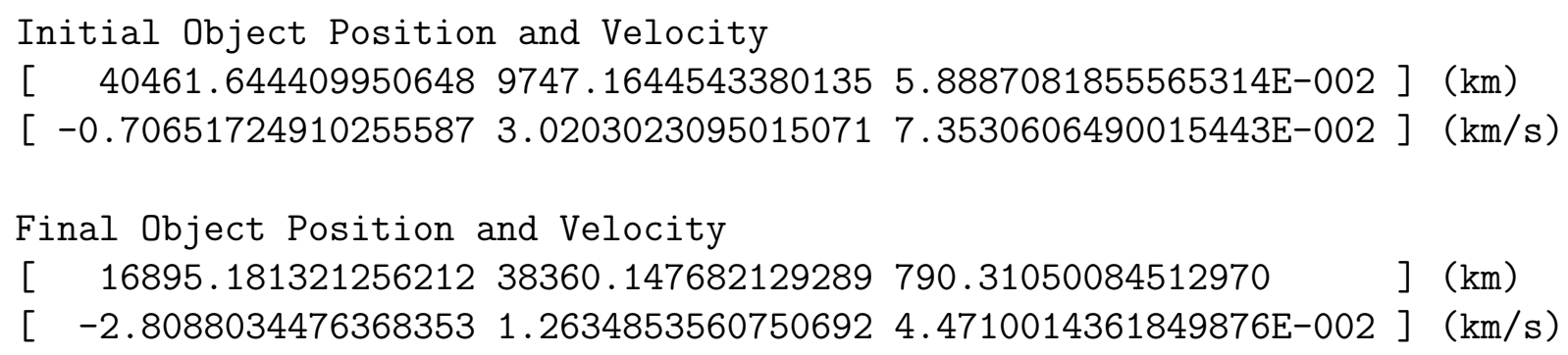




\subsubsection{User Input}

Upon execution, CDPROP will prompt the user for a TLE file along with other propagation parameters. The following section describes each input parameter.

\section{- TLE file}

A TLE in a text file which includes the satellite name and two lines of orbital information is specified as a string into CDPROP's Terminal interface. An example file is shown below:

Breeze-M R/B 32375 2007-058C

132375 U 07058C 08001.61109714 -.00000146 00000-0 10000-3 000018

$232375000.1214088 .43450253571020 .4368 \quad 253.9016 \quad 01.0442978289$

\section{- Propagation time}

Propagation time is given as a real number value in days.

\section{- Spacecraft Mass}

Spacecraft mass is required for calculating the solar radiation pressure perturbing acceleration. This real number parameter should be given in kilograms. The default setting is $1000 \mathrm{~kg}$.

\section{- Spacecraft Area}

Spacecraft area facing the sun is required for calculating the solar radiation pressure perturbing acceleration. This real number parameter should be given in $\mathrm{m}^{2}$. The default setting is $20 \mathrm{~m}^{2}$.

\subsubsection{Output}

CDPROP outputs a text file with the object's position and velocity vector as well as another text file with the object's COEs at each time step. This file can easily be 
uploaded into MATLAB for visualization purposes.

\subsection{Verification and Validation}

In order to continue the use of CDPROP, verification and validation was a major step in this thesis. It was verified using STK simulations to ensure that it was coded properly, and then it was validated to see how accurately the model reflected the truth using objects of interest. Because any propagator can coincidentally produce the location of the satellite at certain instants of time, an ephemeris, or a set of state vectors over time, is used instead. This ensured the average residual behavior of the propagation routine is found [12].

\subsubsection{Verification Using Titan}

Using STK for the verification case, a 10 day propagation with 0.01 day time steps (using 4 x 4 gravity, spherical solar radiation pressure with a coefficient of reflectivity of 1.1, and lunar and solar body effects) was used to verify that the propagator was coded correctly. Figures 5.5, 5.6, and 5.7 show the results. From Figure 5.5, it is verified that CDPROP does mimick the general behavior of the Titan debris object over a 10 day span. This however does not depict the true results of the simulation. Another important aspect that needed to be studied was the residuals over time. 


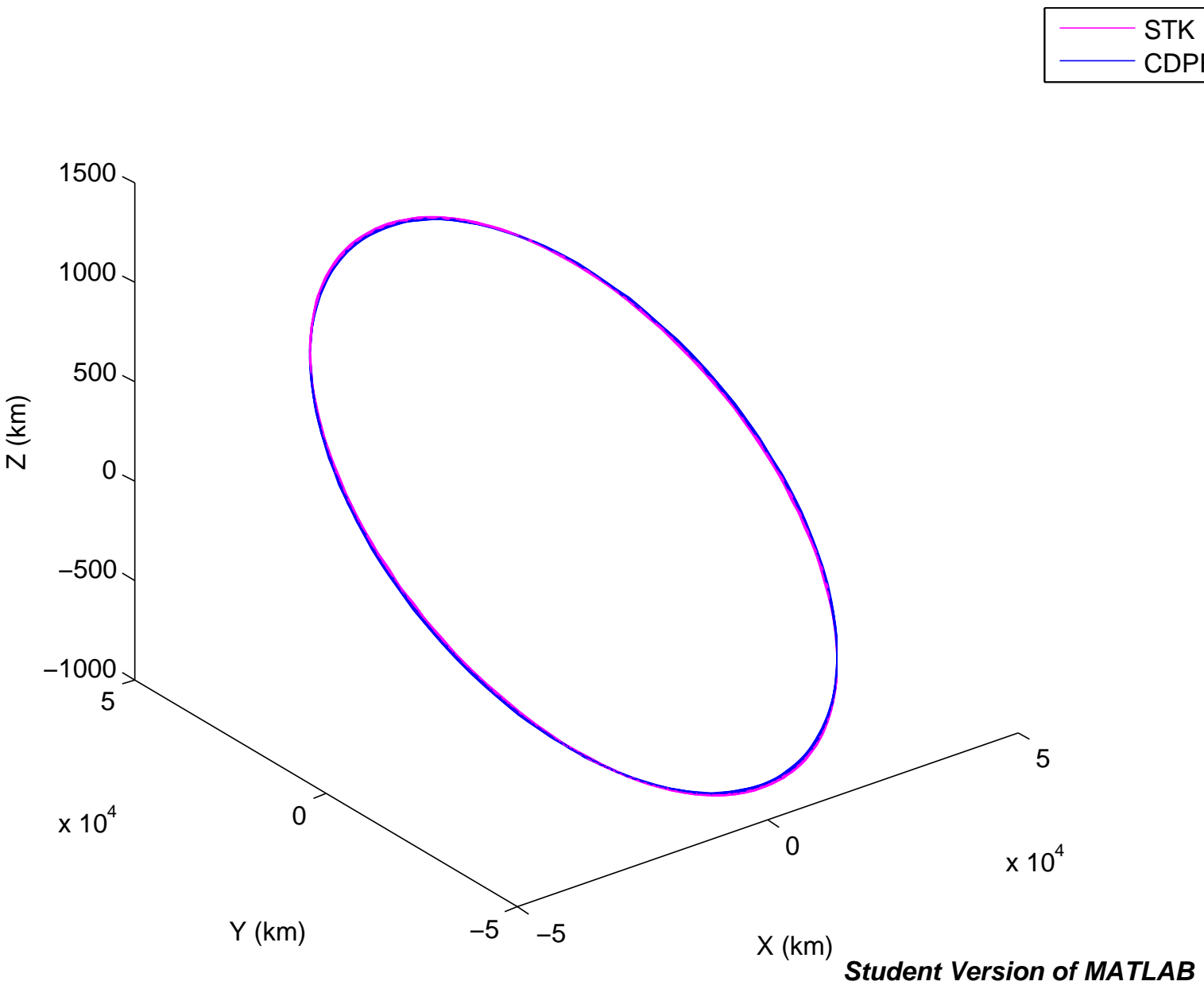

Figure 5.5: An overview of the orbital path of STK's simulation and CDPROP's simulation. STK is in blue while CDPROP is in magenta.

The residuals were calculated using the following,

$$
\hat{y}=y_{o}-y_{c}
$$

where $\hat{y}$ is the residual, $y_{o}$ is the observed point, and $y_{c}$ is the calculated point [12]. The residuals are shown in Figures 5.6 and 5.7 and display a general sinusoidal residual behavior that increases over time. 

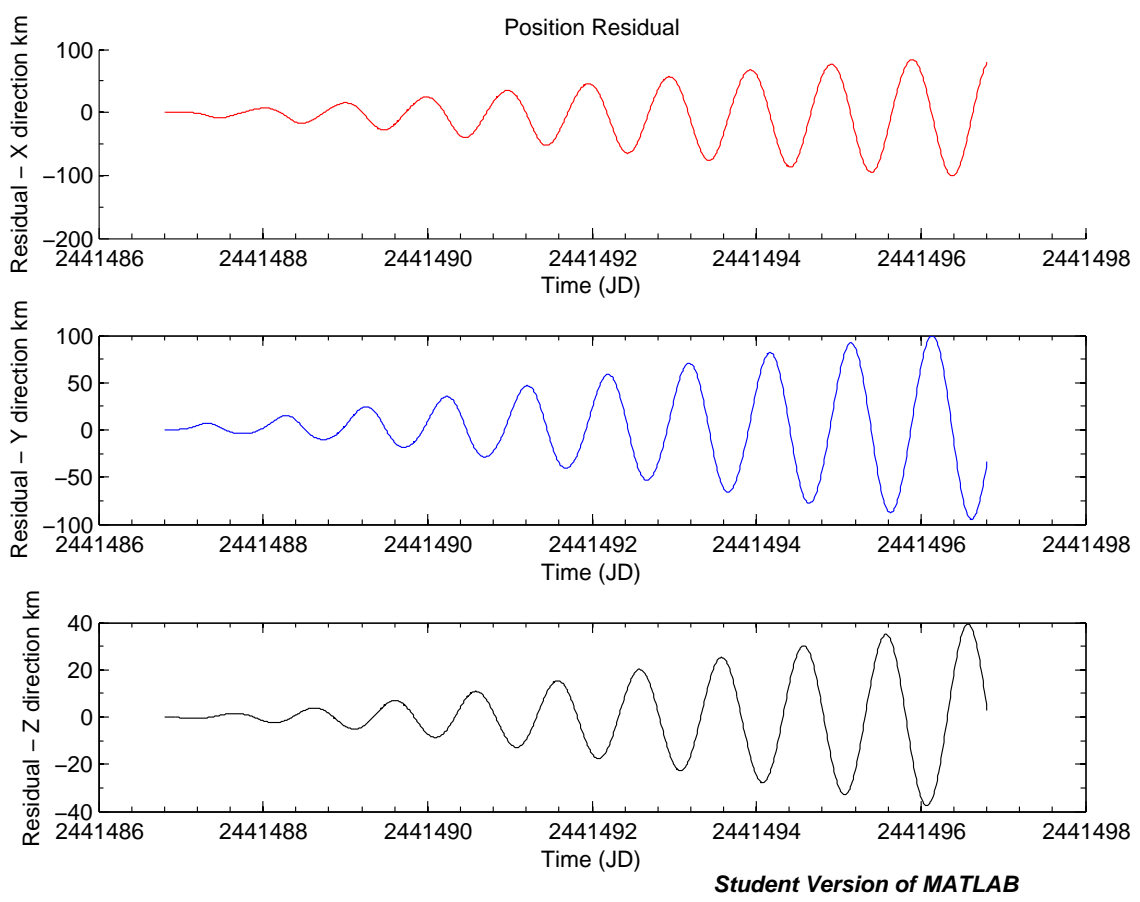

Figure 5.6: Position residuals over 10 days are shown.
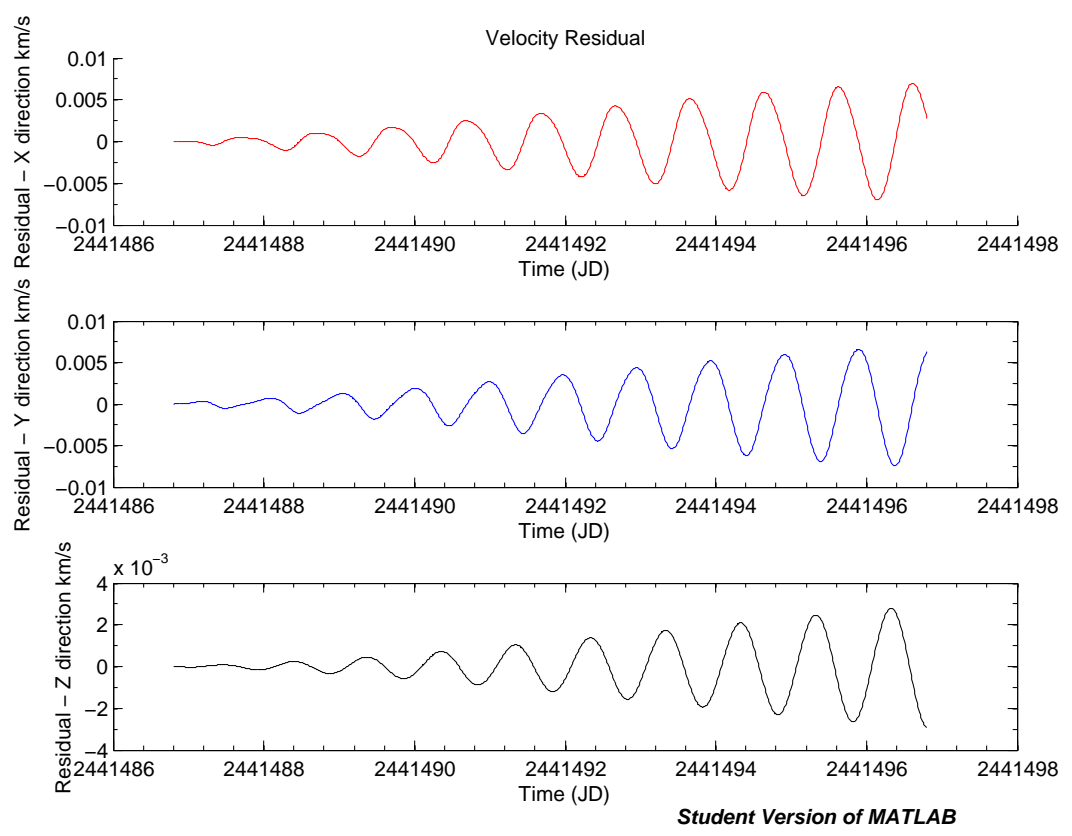

Figure 5.7: Velocity residuals over 10 days are shown. 
The increasing error over time was found to be mainly due to the differences in integration and different methods of obtaining certain parameters in the propagation. STK documentation unfortunately did not give specifics as to its integration method or propagation assumptions; however, CDPROP used a Runge-Kutta Felhberg integration method in Cowell's method for propagation. Over time, this is the main explanation for the differences. Other smaller reasons could be due to ephemeris. STK uses JPL ephemeris directly in its calculations of the sun and moon. CDPROP uses the Astronomical Almanac's algorithm for estimating the positions based on Julian date. Despite these differences, CDPROP displays satisfactory behavior in propagating GEO satellites and objects compared to STK. It is with this that CDPROP's verification passes its requirements and ensures that it is coded correctly within its means. From verification, CDPROP was next passed through validation.

\subsubsection{Validation using Selected TLEs}

CDPROP's validation was based off historical TLE data of three classes of objects.

- Drifting object - Applications Technology Satellite (ATS) 5

- East object - Fengyun 2D

- West object - Skynet 1

- E-W object - OPS 9432 (DSCS 2-2)

These objects were propagated for 10 years using CDPROP and the following parameters: 0.01 day time step, 16 x 16 gravity model, and propagation time of 3652.5 days, or 10 years. Each object had its own estimated $\mathrm{A} / \mathrm{m}$ ratio used in the analysis. In order see the differences between the objects visually, Figures 5.8, 5.9, and 5.10 were created. 


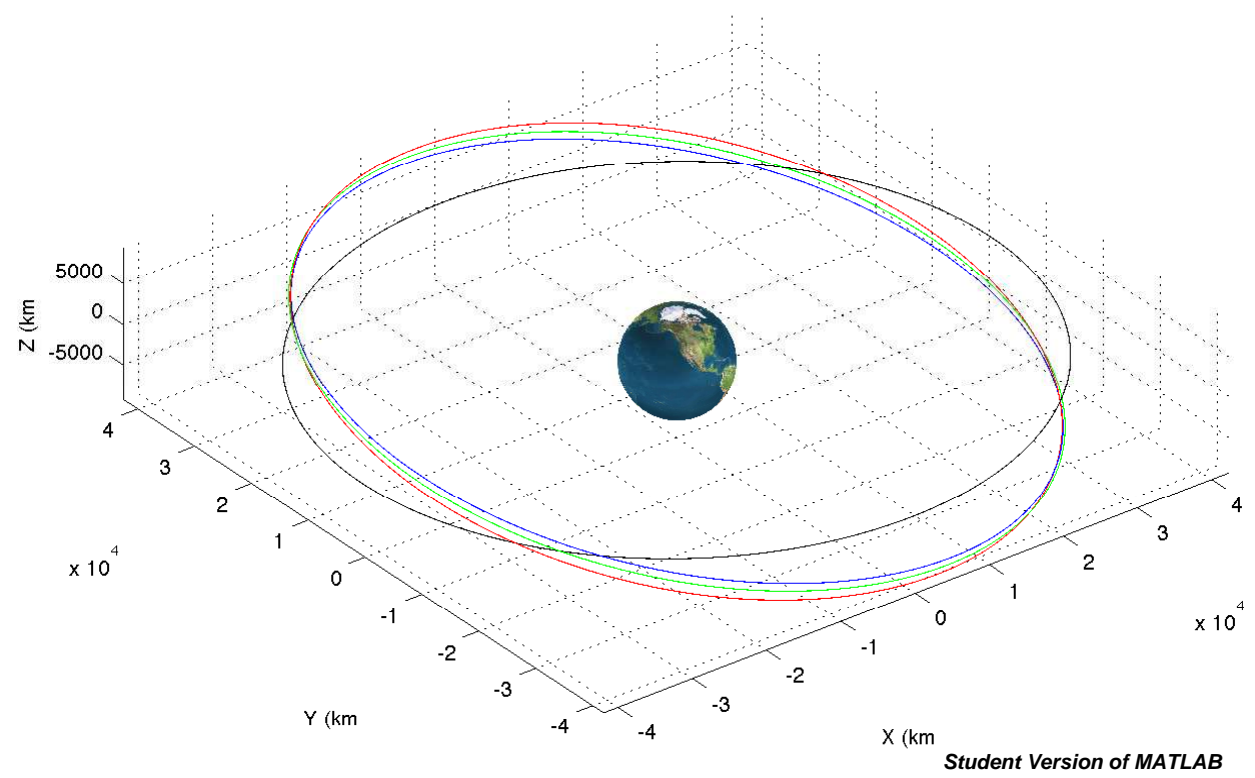

Figure 5.8: Comparison of four objects' orbits over a few periods. The red trajectory is Skynet 1. The blue trajectory is OPS 9432. The black trajectory is Fengyun 2D and the green is ATS 5.

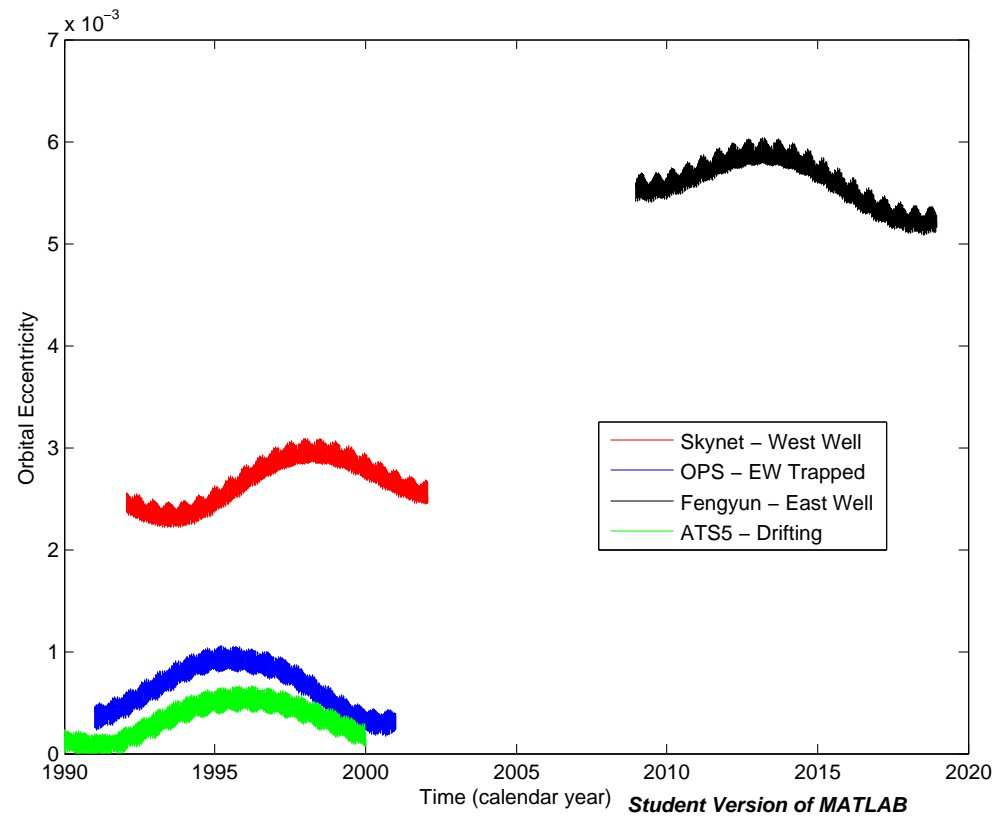

Figure 5.9: Comparison of eccentricity between objects shows Fengyun has the highest eccentricity while ATS5 has the lowest. 


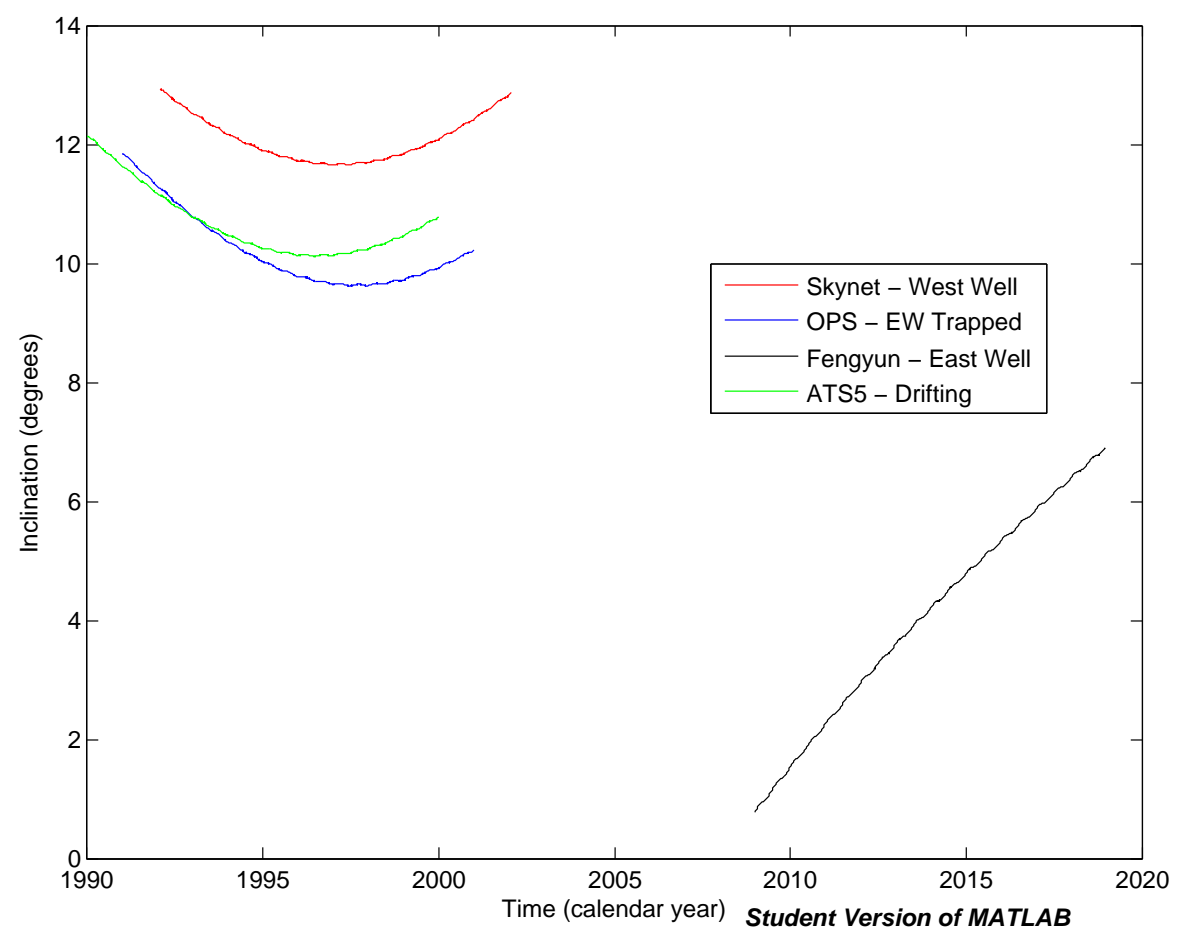

Figure 5.10: Comparison of inclination between objects shows similar behavior for Skynet, OPS, and ATS 5; however, Fengyun changes inclination quite differently.

\section{ATS 5}

ATS 5, NORAD ID 4068, is a US satellite launched on August 12, 1969 for NASA. It has a perigee of $35,990.0 \mathrm{~km}$ and apogee of $36,034.1 \mathrm{~km}$ with a $10.1^{\circ}$ inclination. It suffered a partial misison failure due to a failed experiment deployment. It is cylinder shape covered with solar cells and has an estimated $\mathrm{A} / \mathrm{m}$ of 0.00988 . It is not trapped in the either geopotential well. It is known as a drifter. Figure 5.11 shows traditional drifitng behavior in longitude. It oscillates from $0^{\circ}$ to $360^{\circ}$ throughout its orbit. Because this longitudinal behavior followed the tranditional drifter behavior, it was confirmed that CDPROP could properly model a drifting GEO object. 


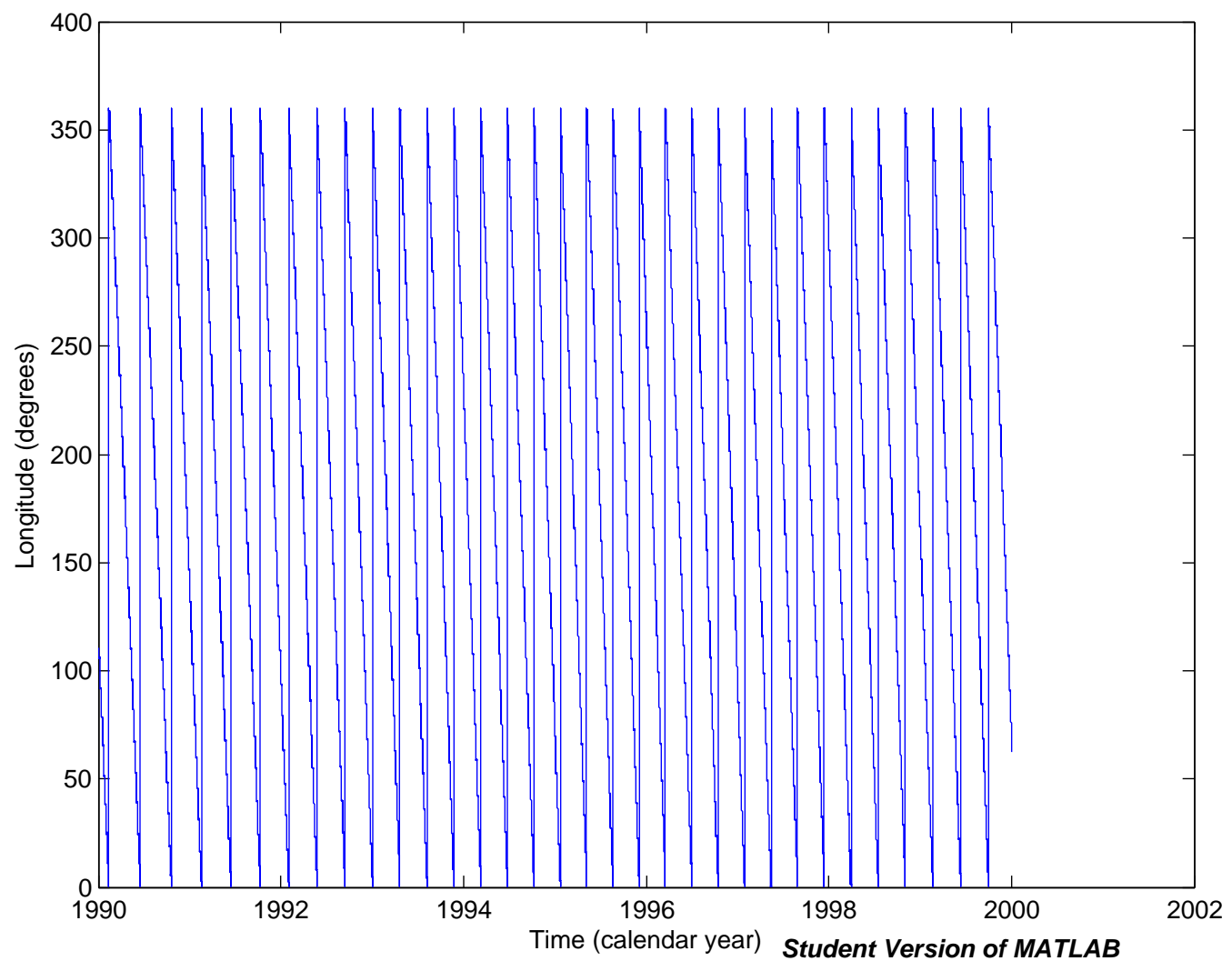

Figure 5.11: Longitudinal history of ATS 5 depicts characteristic drifter behavior.

\section{Fengyun 2D Debris Piece}

The Fengyun 2D debris piece, NORAD ID 33458, originated from the People's Republic of China in 2006. It has a perigee of $35,475.7 \mathrm{~km}$ and apogee of $36,125.6$ $\mathrm{km}$, along with an $3.1^{\circ}$ inclination. It is currently trapped in the $75^{\circ} \mathrm{E}$ well. It is thought to be a small debris piece released in GEO, perhaps a sensor cover. It has an estimated $\mathrm{A} / \mathrm{m}$ ratio of 0.0714 . Figure 5.12 shows the longitudinal beahvior of the object over the 10 year simulation. Compared to Figure 5.11, it can be seen that the Fengyun 2D object is bounded by some longitude bin over time. This is evidence that CDPROP is observing the presence of the Eastern $75^{\circ}$ well. 


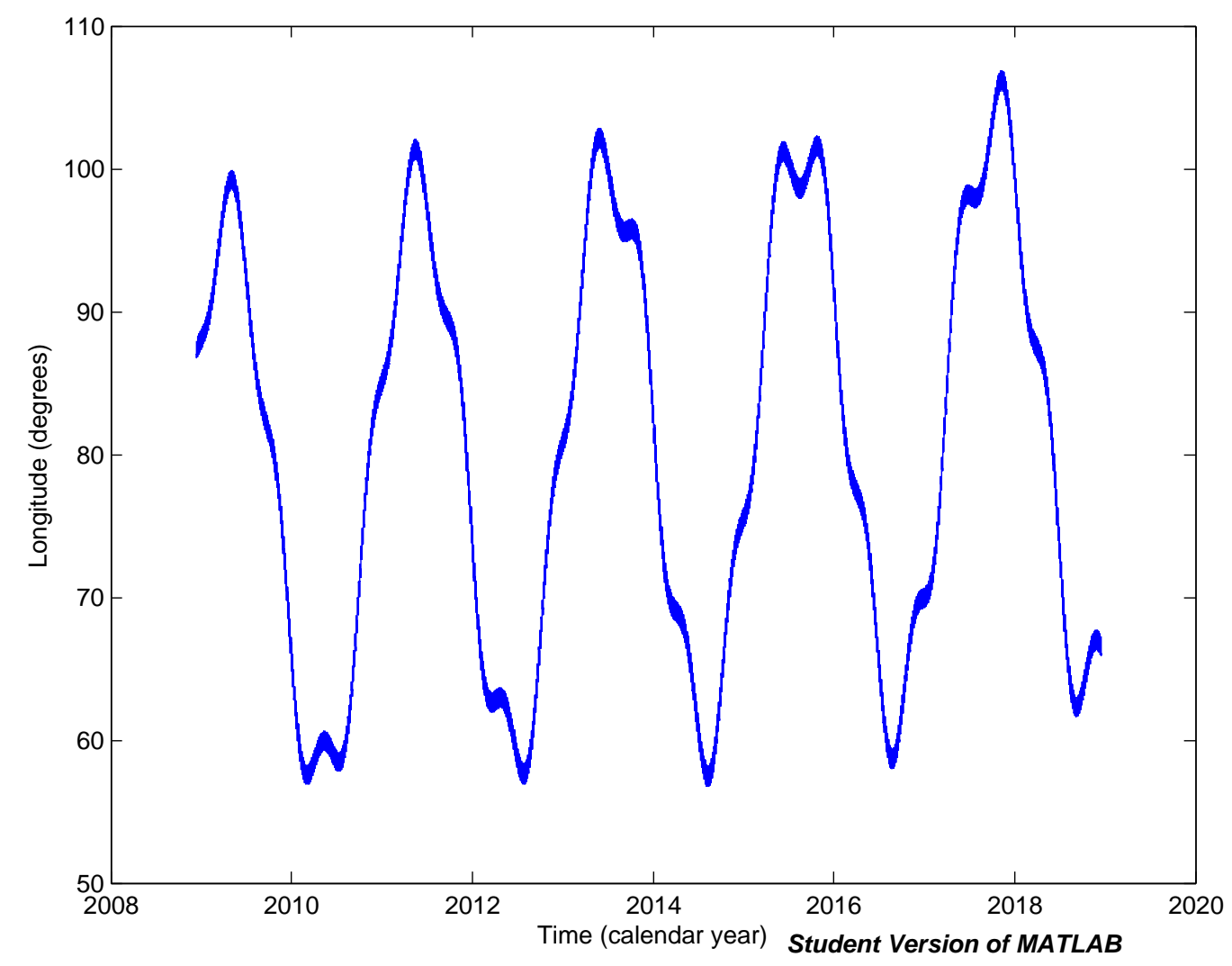

Figure 5.12: Longitudinal history of Fengyun 2D depicts characteristic trapped behavior, in this case the Eastern geopotential well.

\section{Skynet}

Skynet 1D, NORAD ID 4250, is a UK satellite launched on November 22, 1969. It has a perigee of $35,683.8 \mathrm{~km}$ and apogee of $35,901.6 \mathrm{~km}$ with a $9.6^{\circ}$ inclination. It is currently trapped within the Western geopotential well. Figure 5.13 shows the longitudinal behavior of the object over the 10 year simulation using CDPROP. Compared to Figure 5.11, it can be seen that the Skynet 1 object is bounded by some longitude bin over time. This is evidence that CDPROP is observing the presence of the Eastern $105^{\circ} \mathrm{W}$ well, which would be $255^{\circ}$ on the figure. 


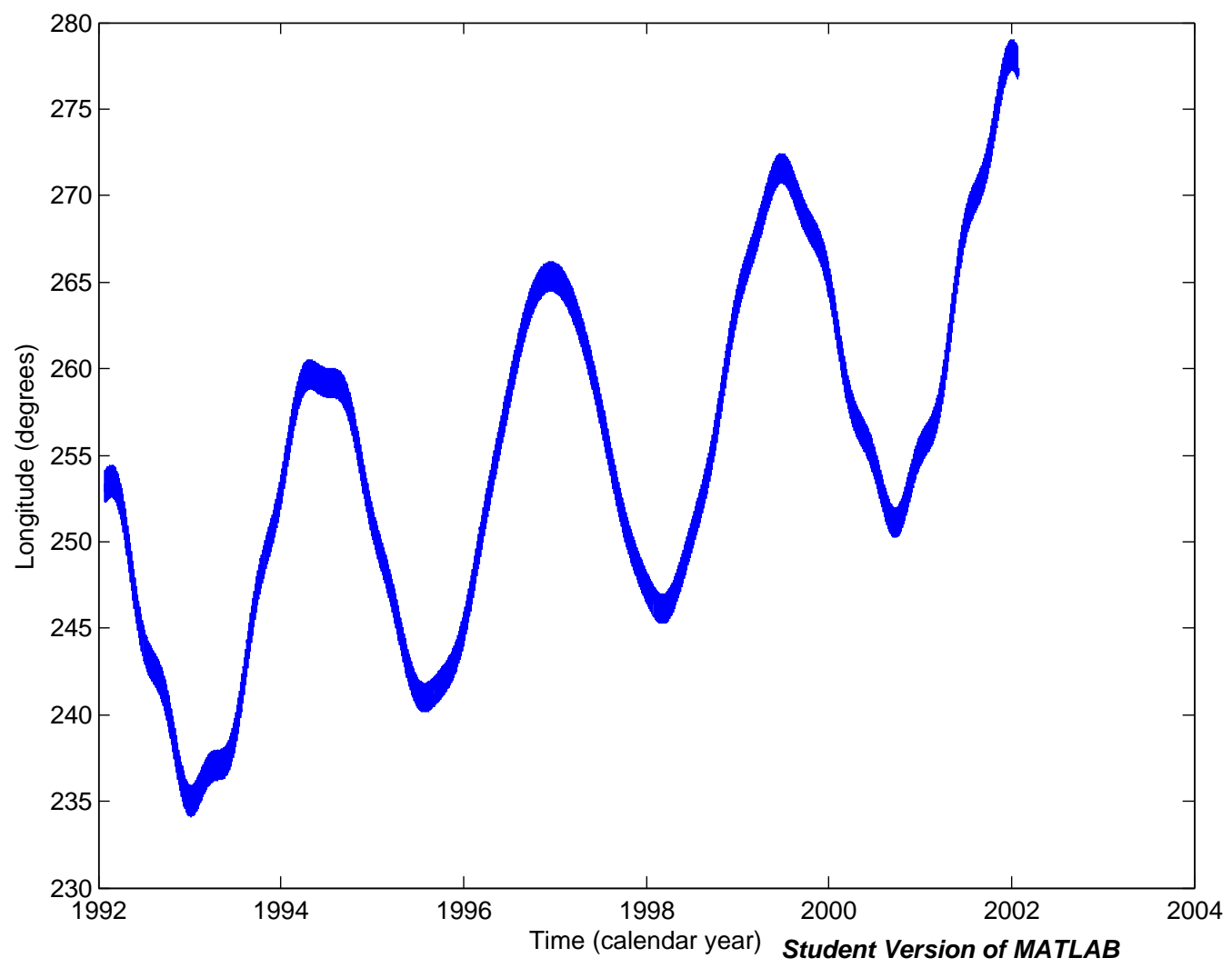

Figure 5.13: Longitudinal history of Skynet 1 depicts characteristic trapped behavior, in this case the Western geopotential well.

\section{OPS 9432 (DSCS 2-2)}

OPS 9432, NORAD ID 5588, is a US satellite launched November 3, 1971 that failed after less than a year of operation.It has a perigee of $35,786.8 \mathrm{~km}$ and an apogee of $35,813.8 \mathrm{~km}$, with a $11.5^{\circ}$ inclination. It has a $\mathrm{A} / \mathrm{m}$ ratio estimate of 0.0128 and has a cylindrical body shape. It is currently trapped between the East and West wells, oscillating between them. Based on the 10 year simulation, CDPROP was not able to capture the oscillations even by increasing the gravity model to a $18 \mathrm{x} 18$. The underlying issue could be due to the method of determining the geocentric longitude. There are several methods available which offer higher accuracy; however the chosen one assumed longitude to be equivalent to right ascension. The OPS 9432 satellite 
according to ESA's DISCOS database, which uses historical data, should be bound from $190^{\circ}$ to $540^{\circ}$, right in between the East and West wells as shown in Figure 5.15.

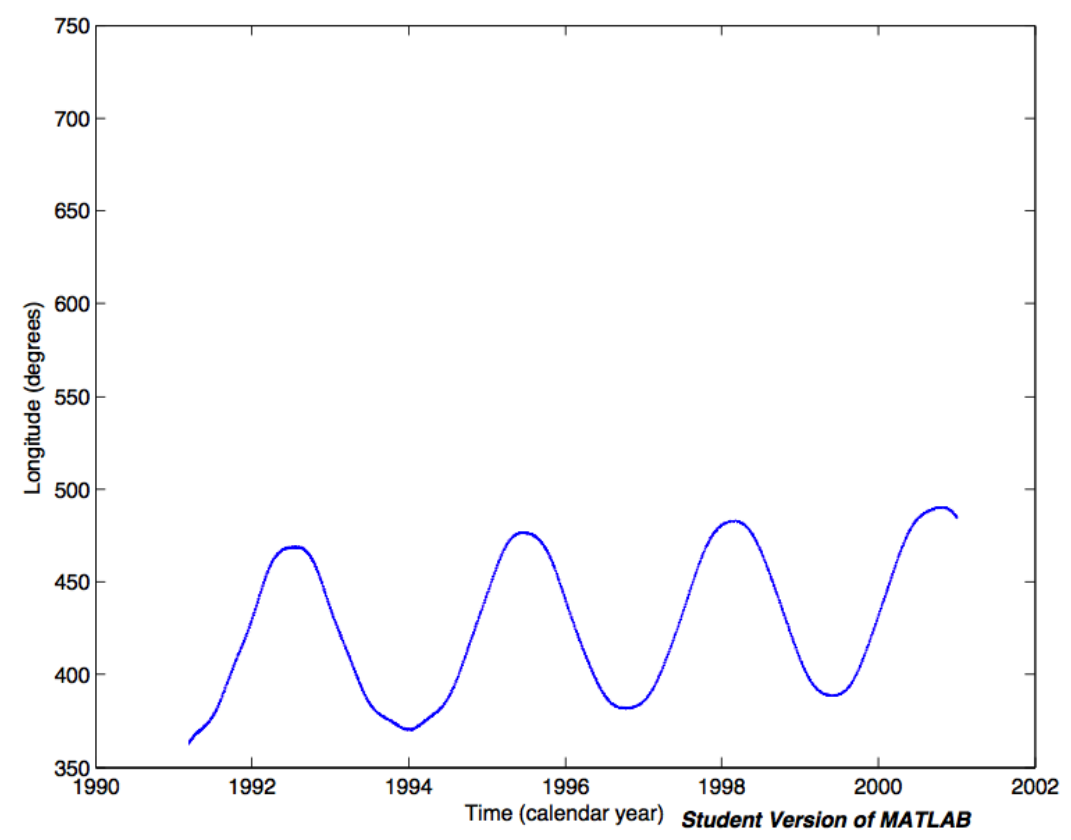

Figure 5.14: Longitudinal history of OPS 9432 does not show the characteristic behavior of EW trapped objects.

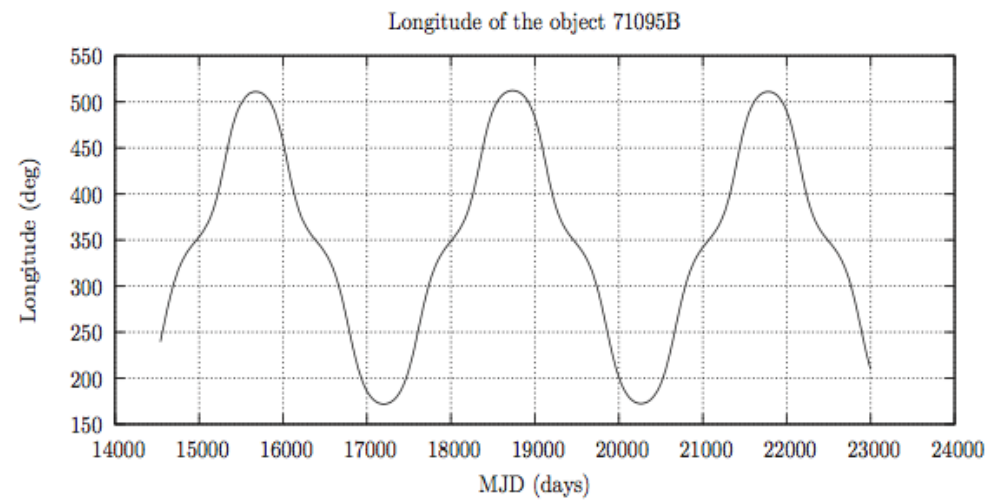

Figure 5.15: The OPS 9432 satellite according to ESA's DISCOS database should be bound from $190^{\circ}$ to $540^{\circ}$, right in between the East and West wells. 


\subsubsection{Residuals from Historical Data}

Previously in verification, CDPROP was compared with STK. This only was a test in order to see if CDPROP was coded correctly and is only a theoretical simulation, as Titan was an object that actually exploded on orbit. It was assumed that it was an intact satellite. An important aspect in developing an orbit propagator is to understand how close it comes to the truth. It is important to note that very few propagators can get close to the truth; however, understanding the limitations of the propagator is crucial. For this, historical data must be used. Historical TLE data for the ATS 5 object was used to visually see the residuals as shown in Figure 5.16. ATS 5 was chosen for looking at the residual since it was a general drifting satellite. From Figure 5.16, it can be seen the the $\mathrm{X}$ and $\mathrm{Y}$ direction have the largest amount of error over time, while the $\mathrm{Z}$ direction shows the least amount of error. It should be noted

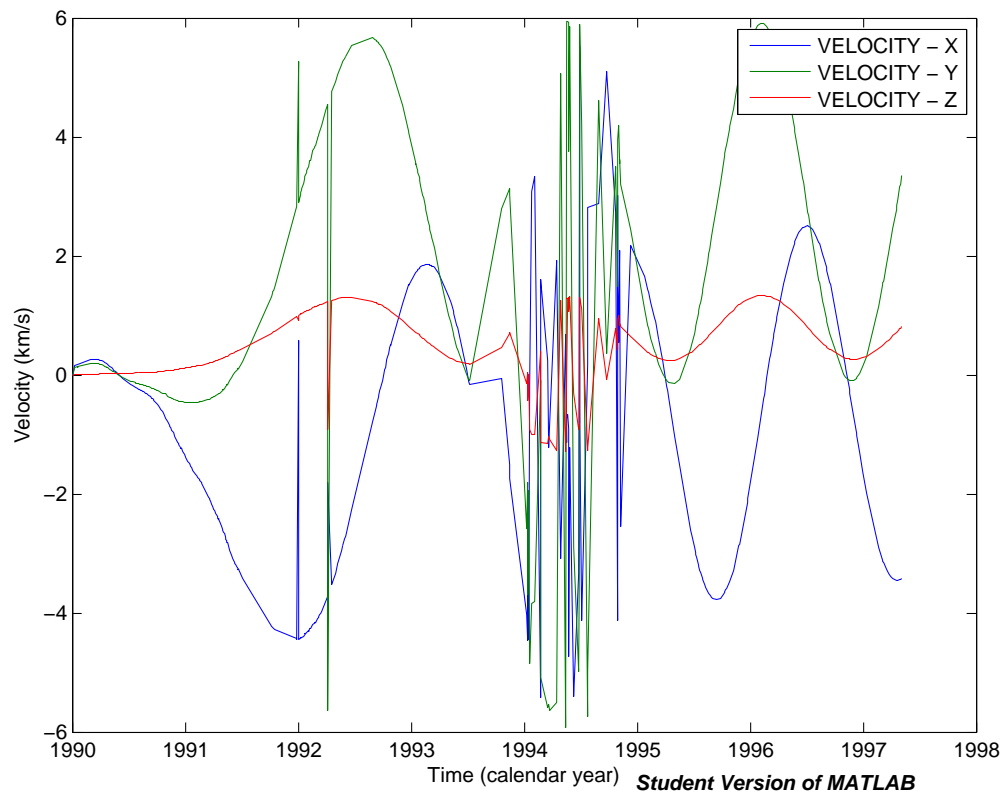

Figure 5.16: Comparison of ATS 5's historical data and CDPROP's simulation.

that there were several issues with the TLE formatting in the historical data from the 
database, which can be common. It is thought that the mean motion was the cause for the issue based on Figure 5.17. It was attempted to remove the badly formatted TLEs; however, there were still some found throughout the data set. Points were the first derivative of the mean motion was suspect were removed; however, there were patterns of bad data which can be seen in Figure 5.17.

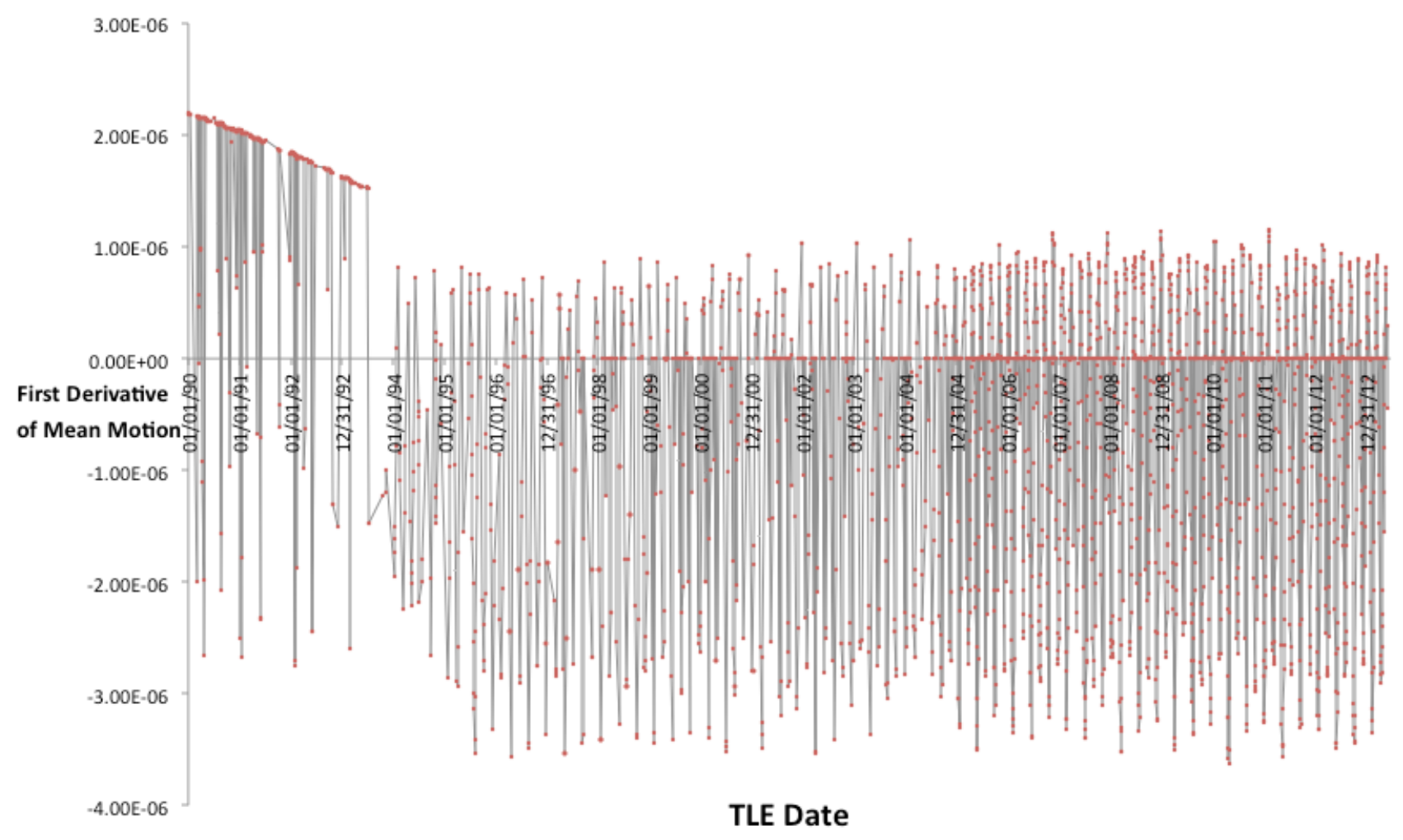

Figure 5.17: A generation of the first derivative of mean motion values showing suspected values.

\subsubsection{Conclusion}

CDPROP does well in predicting the longitudinal behavior of Eastern, Western, and drifting objects; however, it does not do well in catching objects that are trapped in the Eastern-Western wells. Further work must be done to see if objects trapped in Eastern-Western wells may be more sensitive to errors in predicting historical 
behavior. CDPROP had significant differences with historical data in the $\mathrm{X}$ and $\mathrm{Y}$ direction using the ATS 5 debris piece. Future study may be needed to inquire as to the significant differences. 


\section{Chapter 6}

\section{Application of CDPROP: Collision Modeling}

One of the main advantages CDPROP offers is the utility it creates in studying objects in GEO. CDPROP has a much faster runtime than any MATLAB or other computationally expensive tool. This allows for faster runtimes of simulations. This section will show an application of CDPROP in modeling collisions near geopotential wells and graveyard orbit.

\subsection{The Example Cases}

To study different parameters, three cases were simulated, a graveyard collision, eastern well collision, and western well collision using the same A/m fragment size, for consistency. It was assumed that after the collision no other collisions between fragments occurred. All collisions were propagated 5 years. The goal in mind was to see if these regions can behave like sinks or rather become sources of debris generation. This was done by looking at the fragments longitudinal history over time as well as 
the fragment's orbit. Below are the parameters of the three cases:

- Graveyard Collision

- Location: $\vec{r}=[16660.1,128569.3,11.7] \mathrm{km}, \vec{v}=[-1.76,0.35,-6.5 \mathrm{e}-05] \mathrm{km} / \mathrm{s}$

- Area to Mass Ratio of Fragments: 0.02

- East Well Collision

- Location: $\vec{r}=[13447.0,-39789.5,300.3] \mathrm{km}, \vec{v}=[2.92,0.976,0.034] \mathrm{km} / \mathrm{s}$

- Area to Mass Ratio of Fragments: 0.02

- West Well Collision

- Location: $\vec{r}=[-37205.9,-20023.5,-226.0] \mathrm{km}, \vec{v}=[1.42,-2.629,-0.686] \mathrm{km} / \mathrm{s}$

- Area to Mass Ratio of Fragments: 0.02

\subsection{Results}

To begin, 1a thousand numbers were generated between 0 and 1 . These random numbers were input into an inverse Cumulative Distribution Function (CDF) and represented probabilities. Using Equation 4.14, the correct $\mu, \sigma$ and $\log (A / M)$ or $\chi$ were input into the inverse CDF to follow the $\Delta \mathrm{V}$ distribution presented earlier in this paper [11]. In Figure 6.1, the $\Delta \mathrm{V}$ distribution is presented. This figure agrees with Hanada's work in that the majority of the fragments are between 0 and $10 \mathrm{~m} / \mathrm{s}$. This distribution gives the necessary information for the magnitude of the departing fragments. From these magnitudes, a Gaussian distribution was applied to the collision site. This meant that it was assumed that the log of the fragment velocity direction followed a normal distribution. Using these unit direction vectors 
and magnitudes, the new fragment state velocity vector can be found. This is further shown in Figure 6.2.

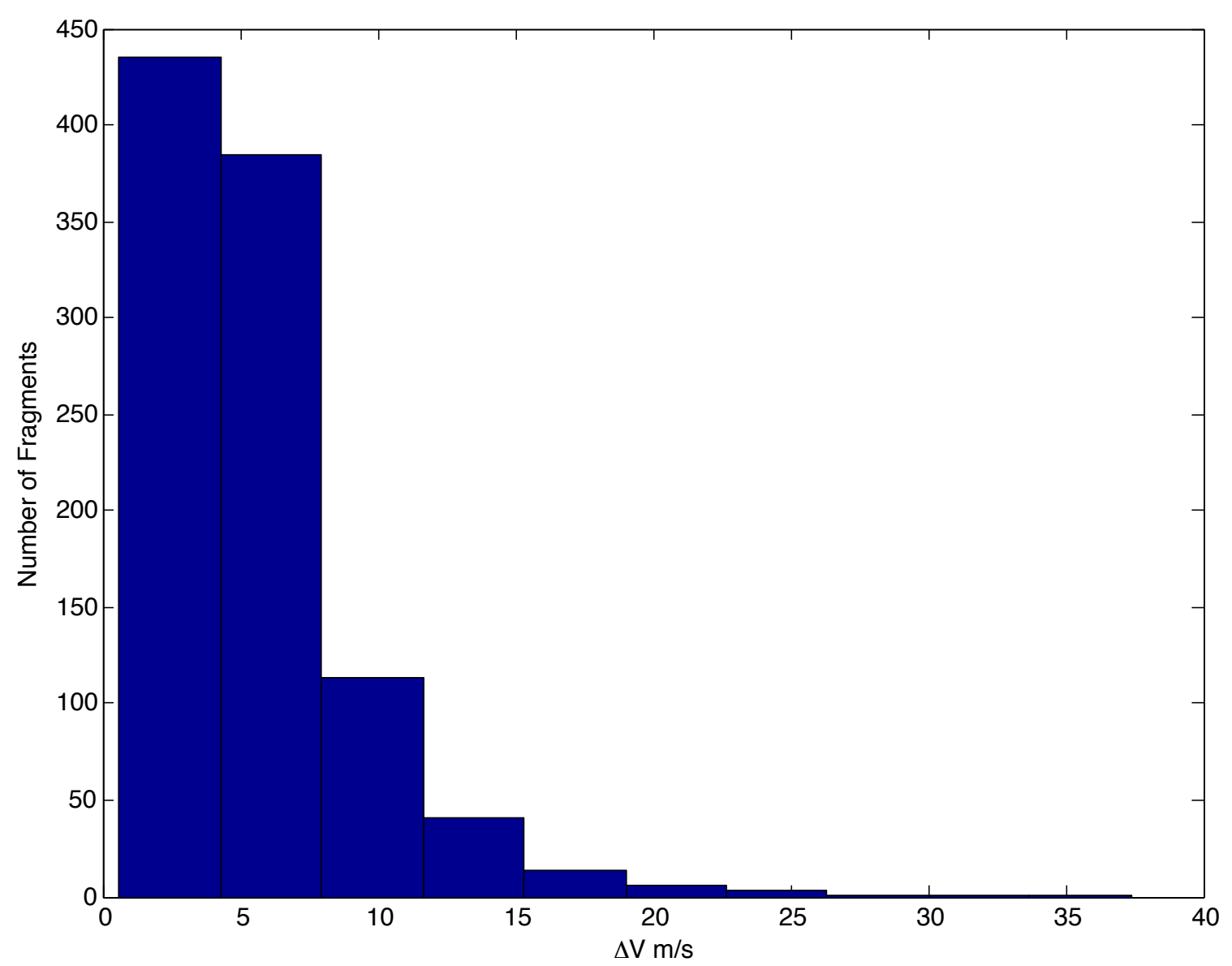

Figure 6.1: $\Delta \mathrm{V}$ distribution shows the majority of fragments ejected are between 0-5 m/s.

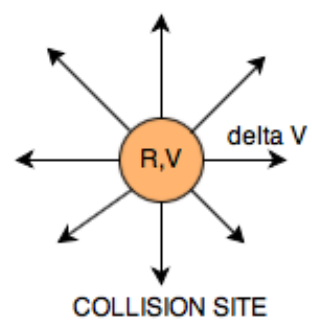

Figure 6.2: This figure depicts the general idea behind finding the fragments new state vectors for propagation. $\Delta \mathrm{V}$ is a product of the distribution magnitude and direction unit vectors. This is then added to the current velocity and the position vector is assumed to be the same, instantaneously. 


\subsubsection{Graveyard Collision Results}

The first case studied was a graveyard collision for the relative simplicity compared to the areas with geopotential wells. Objects in graveyard orbit are thought to act like drifting satellites; however, graveyard is thought to be a "debris" sink due to its distance from any active satellites in the GEO ring. Since the propagation will only be for one year, it should be noted that it is highly unlikely that any debris will come near the GEO ring, as this has been confirmed through work by the NASA Johnson Space Center. This is why graveyard is considered relatively safe for current GEO disposal options. The location of the collision can be seen in Figure 6.3. Figure 6.4 shows

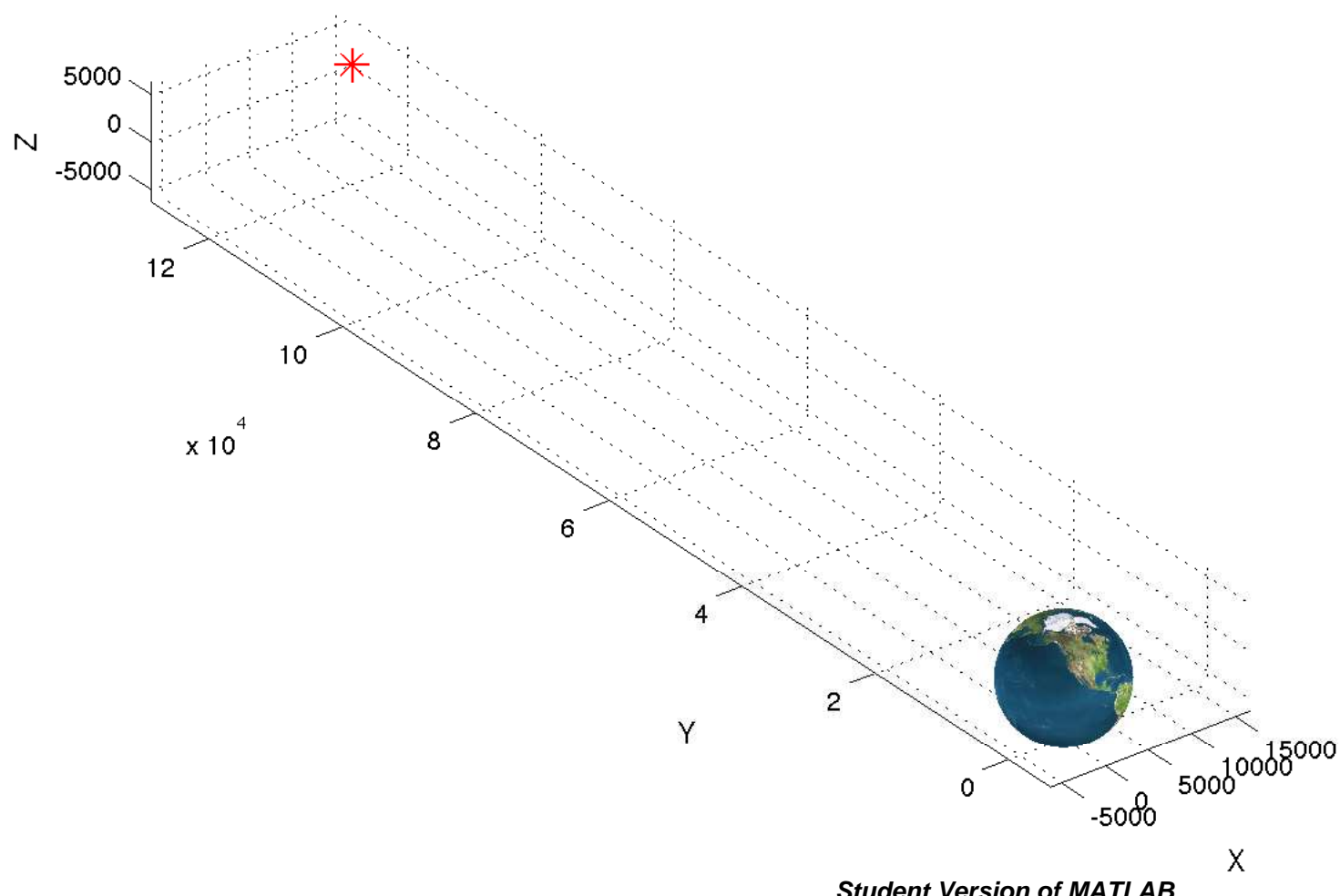

Figure 6.3: A theoretical graveyard collision was simulated in graveyard orbit in this location.

the results of the collision. 1000 fragments were generated using the breakup model; 
however, only the bounds of the fragments were plotted. The magenta orbit represents the slowest imparting debris fragment while the cyan orbit represents the fastest imparting debris fragment. For future reference and clarification, slowest impacting fragments had the smallest $\Delta \mathrm{V}$ applied in the collision whereas the fastest fragments had the largest $\Delta \mathrm{V}$ applied in the collision. It is the same for the cases that follow as well. Just as it was believed before the simulation, no fragments entered near the GEO regime.

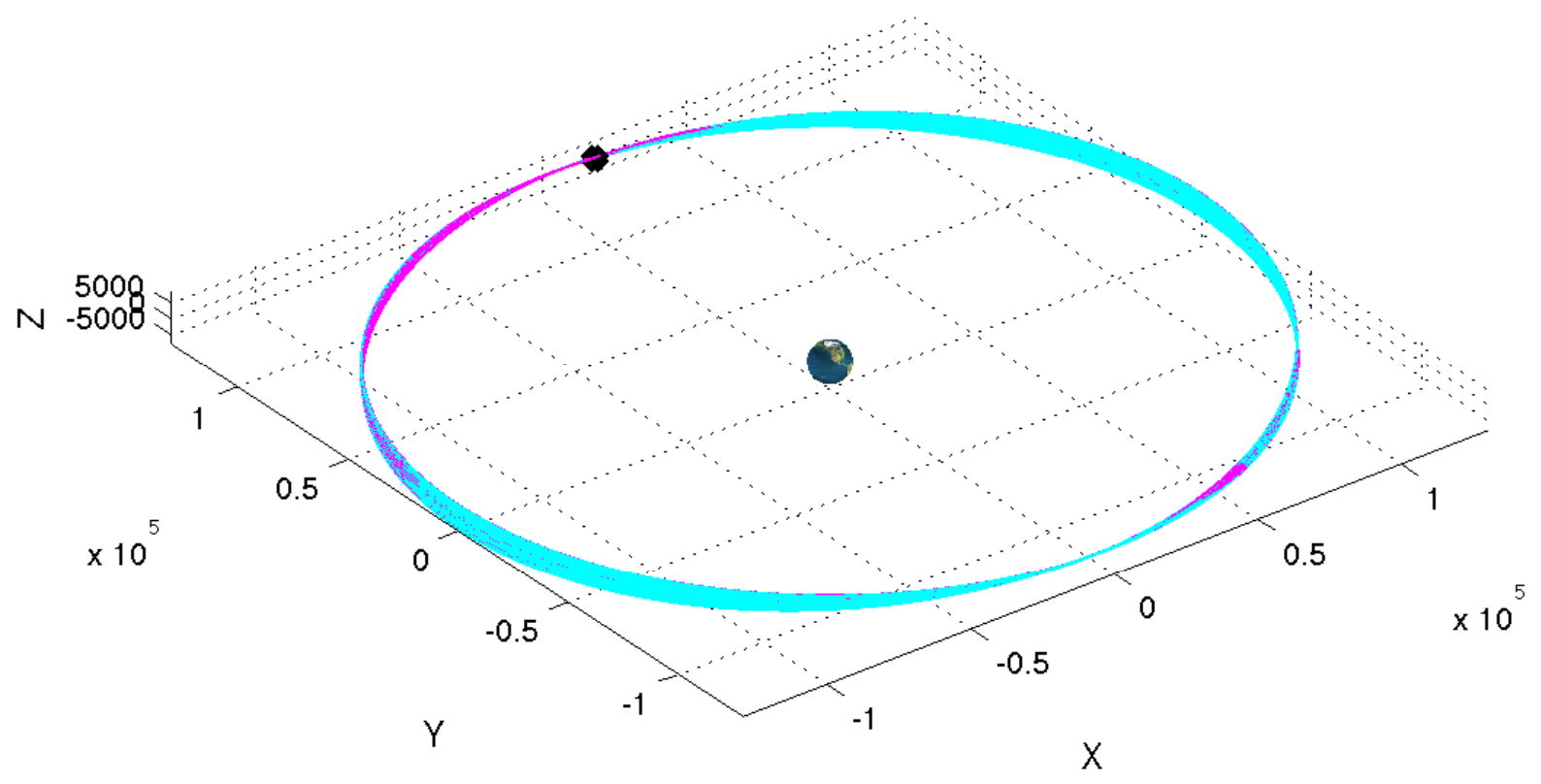

Student Version of MATLAB

Figure 6.4: A theoretical graveyard collision was simulated in graveyard orbit. The magenta orbit represents the slowest imparting debris fragment while the cyan orbit represents the fastest imparting debris fragment. Note the Earth is not necessarily positioned with geographical accuracy. 


\subsubsection{East Well Collision Results}

The second case studied was a collision in the Eastern geopotential well since it is the significantly larger well. Although these areas have yet to be studied in detail, it is thought that objects that become trapped in a well will continuously oscillate about its center forever. This may or may not be the case if internal collisions take place. This collision was simulated for five years since it takes about a few years to see some variation in the longitude, which is the key identifier for trapped objects. The location of the collision can be seen in Figure 6.5.

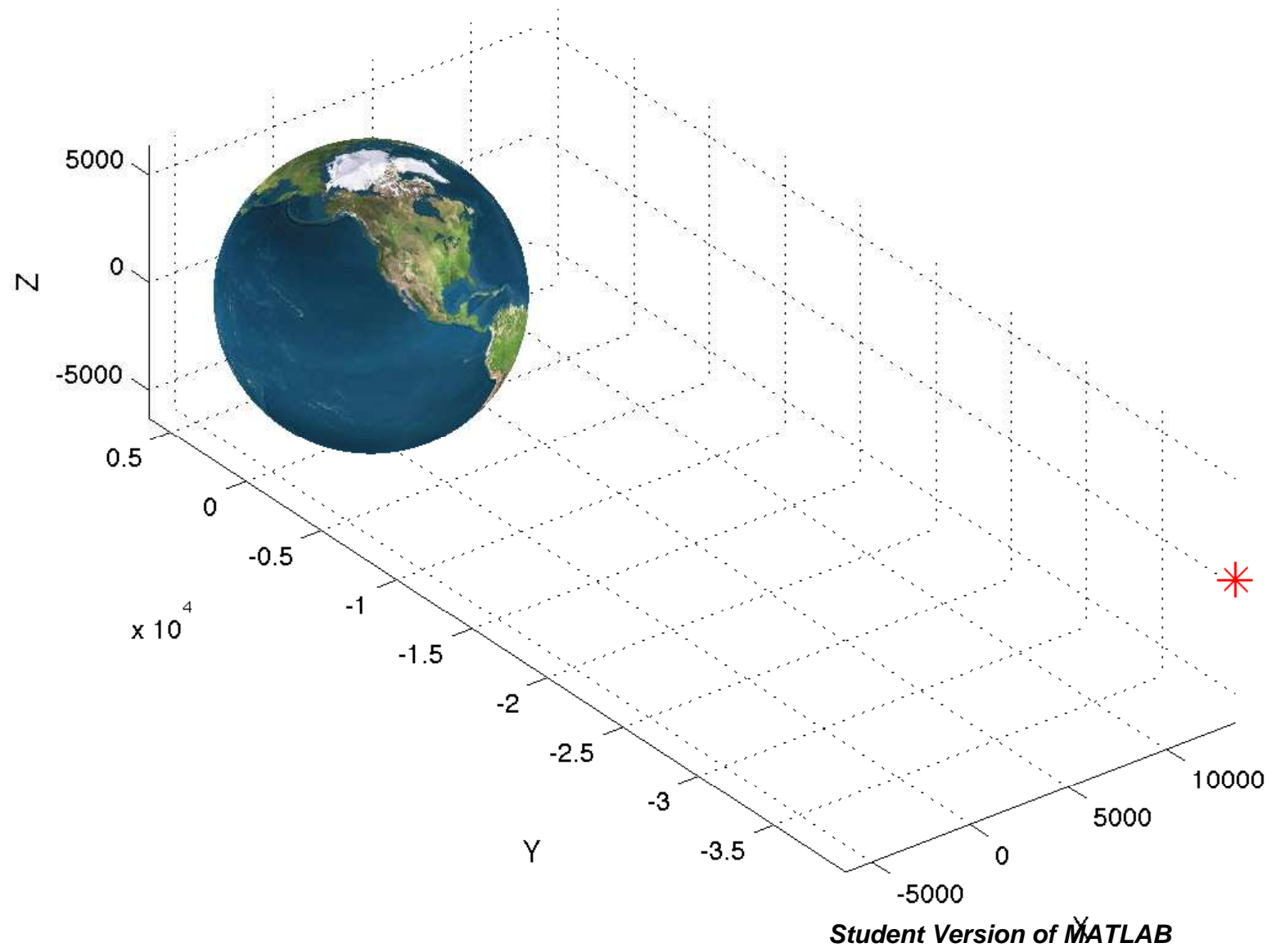

Figure 6.5: A theoretical collision was simulated in an Eastern well. The location is shown. Note the Earth is not necessarily positioned with geographical accuracy. 
The results of simulation are shown in the figures below. Figure 6.6 depicts a year orbit post-collision. The magenta orbit represents the slowest imparting debris fragment while the cyan orbit represents the fastest imparting debris fragment. From the orbit itself, however, it is difficult to know whether or not the objects remained trapped or not. Thus, the longitudinal history over a five year time period is shown in Figure 6.7.

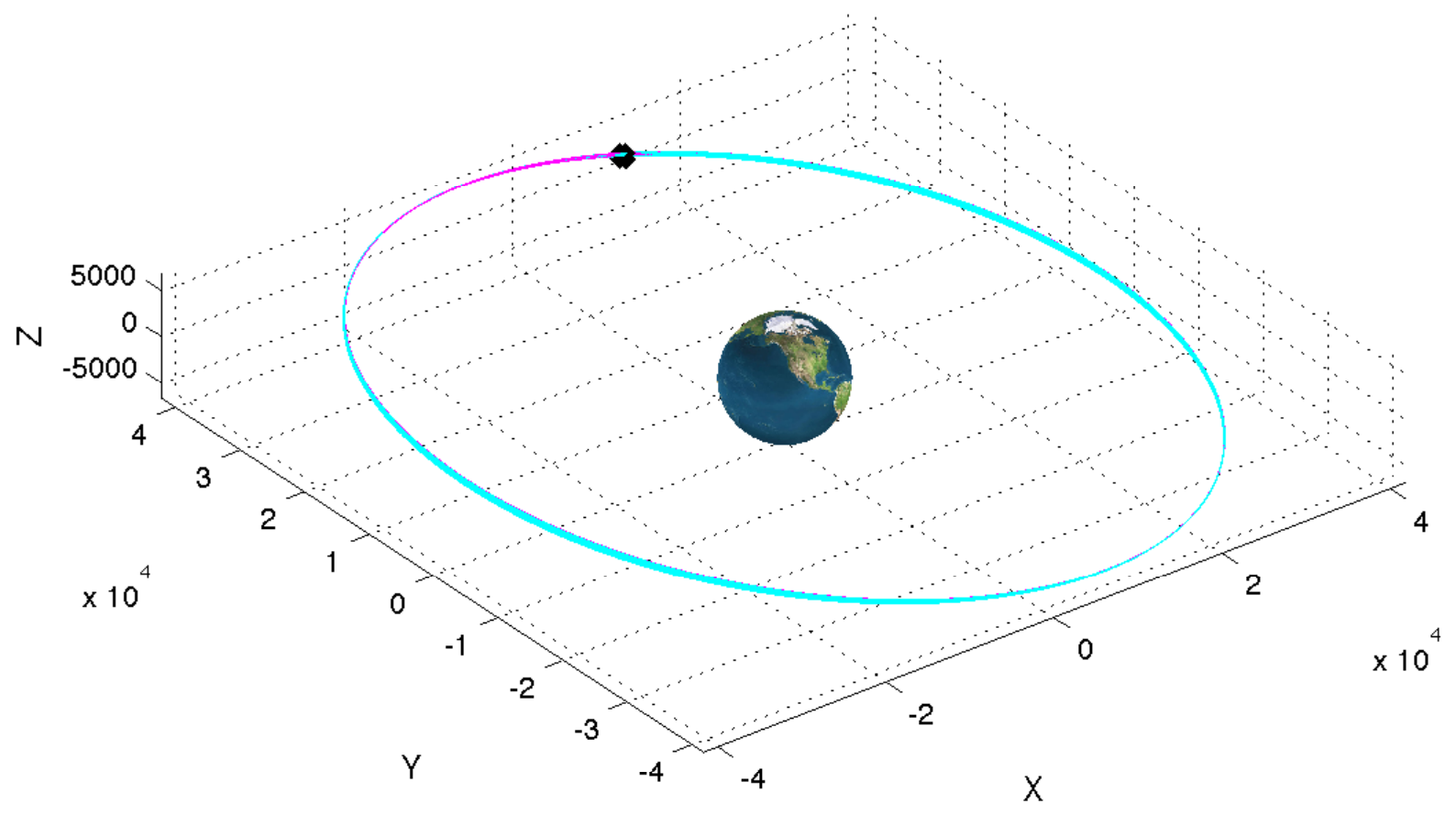

Student Version of MATLAB

Figure 6.6: A theoretical collision was simulated in an Eastern well. The magenta orbit represents the slowest imparting debris fragment while the cyan orbit represents the fastest imparting debris fragment. Note the Earth is not necessarily positioned with geographical accuracy.

The longitudinal history of the Eastern well collision shows interesting behavior. The behavior initially looks as if the fragments go from being East well objects, to being 
East-West trapped objects; however, eventually become drifters. The most likely explanation for this is that these two fragments may have become East-West trapped objects; however, in the validation of CDPROP it was discovered the propagator does not simulate East-West trapped objects well.

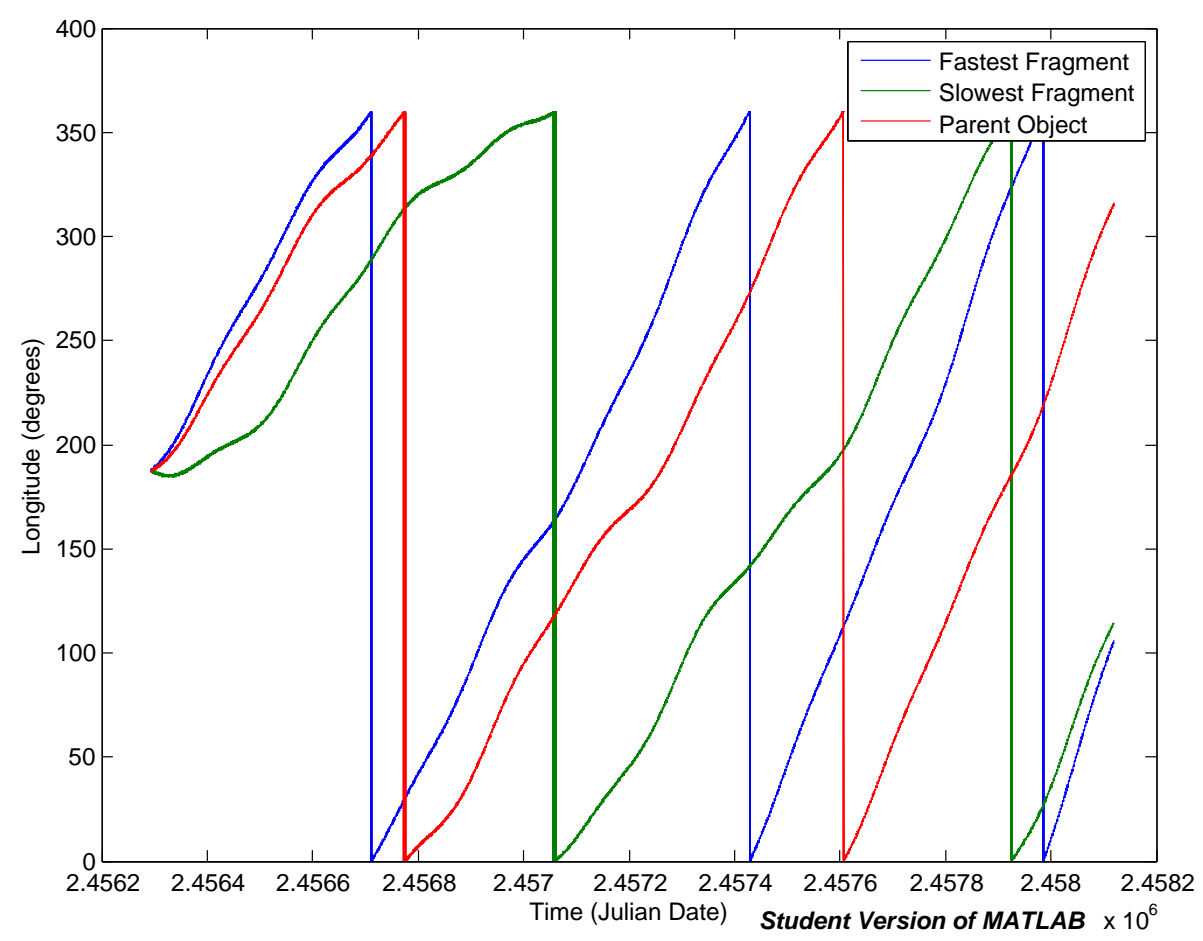

Figure 6.7: The longitudinal history of the collision's slowest and fastest fragments is shown. It initially displays characteristic behavior of a EastWest trapped object but eventually loses that behavior in the simulation.

\subsubsection{West Well Collision Results}

The third case studied was a collision in the Western geopotential well, the smaller of the two wells. As aforementioned, it is thought these regions behave as sinks; however, collisions may or not change these circumstances. Although these areas have yet to be studied in detail, it is thought that objects that become trapped in a well will continuously oscillate about its center forever. This may or may not be the case if 
internal collisions take place. This collision was simulated for five years since it takes about a few years to see some variation in the longitude, which is the key identifier for trapped objects. The location of the collision can be seen in Figure 6.8.

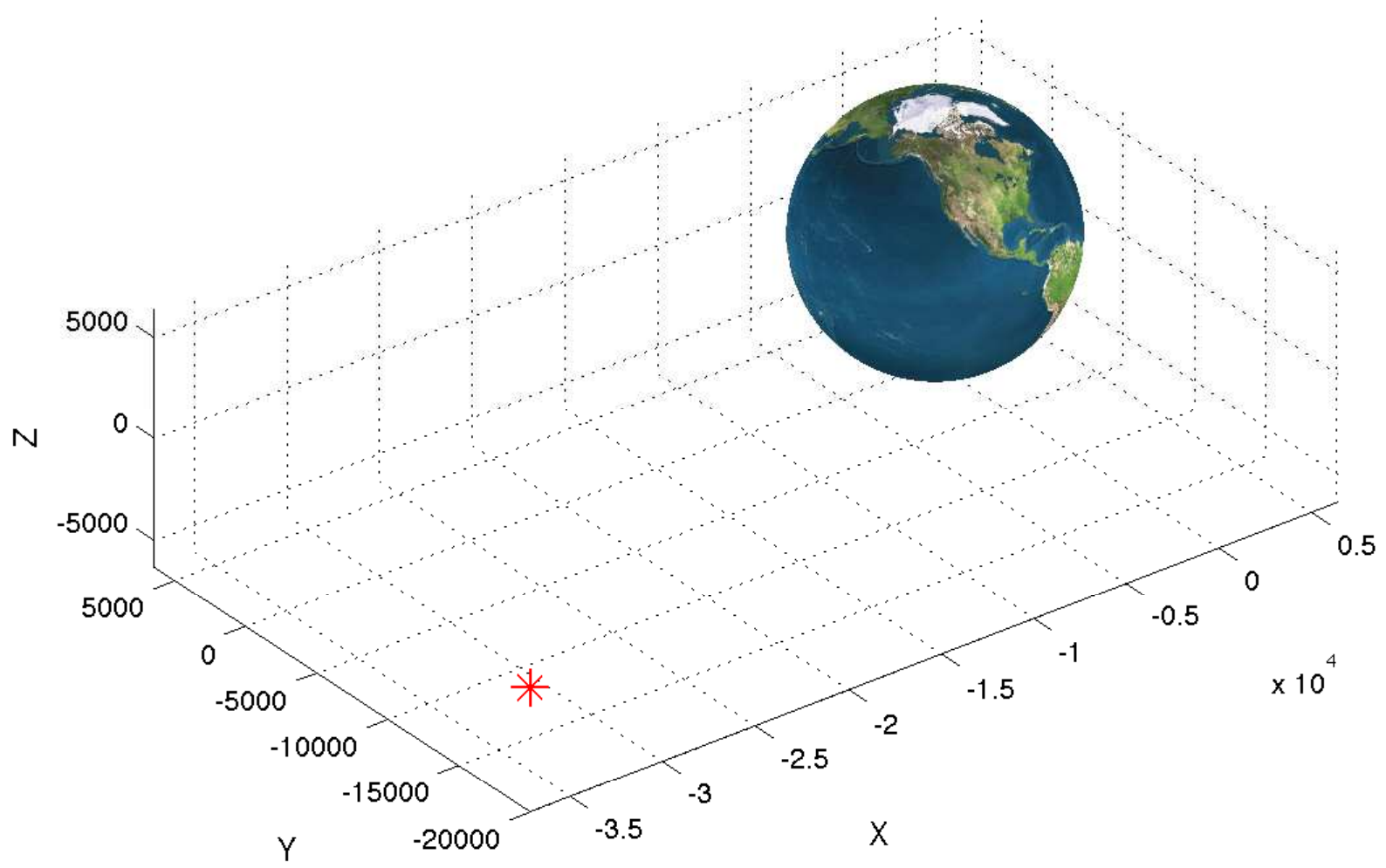

Student Version of MATLAB

Figure 6.8: A theoretical collision was simulated in a Western well. The location is shown. Note the Earth is not necessarily positioned with geographical accuracy.

The results of simulation are shown in the figures below. Similarly to the East well collision simulation, Figure 6.9 depicts a year orbit post-collision. The magenta orbit represents the slowest imparting debris fragment while the cyan orbit represents the fastest imparting debris fragment. From the orbit itself, however, it is difficult to know whether or not the objects remained trapped or not. Thus, the longitudinal 
history over a five year time period is shown in Figure 6.10.

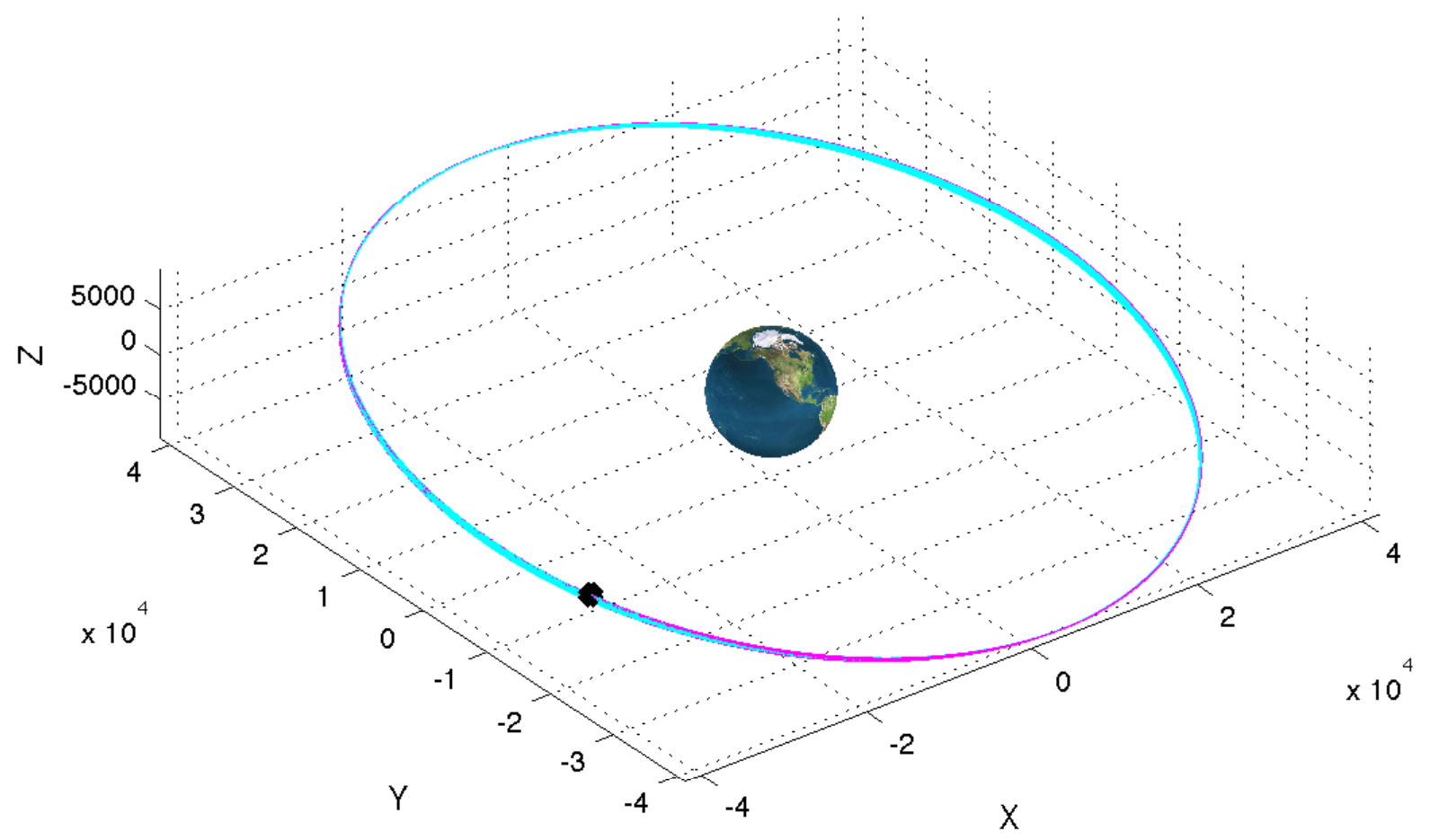

Student Version of MATLAB

Figure 6.9: A theoretical collision was simulated in a Western well. The magenta orbit represents the slowest imparting debris fragment while the cyan orbit represents the fastest imparting debris fragment. Note the Earth is not necessarily positioned with geographical accuracy.

The longitudinal history of the Western well collision shows interesting behavior. The behavior of the fastest fragment and slowest fragment is significantly different from each other. The slowest fragment appears to stay within the West well due it being bound between $100^{\circ}$ and $330^{\circ}$; however, the fastest fragment exhibits drifting behavior and appears to not be trapped based on it's longitudinal variation. 


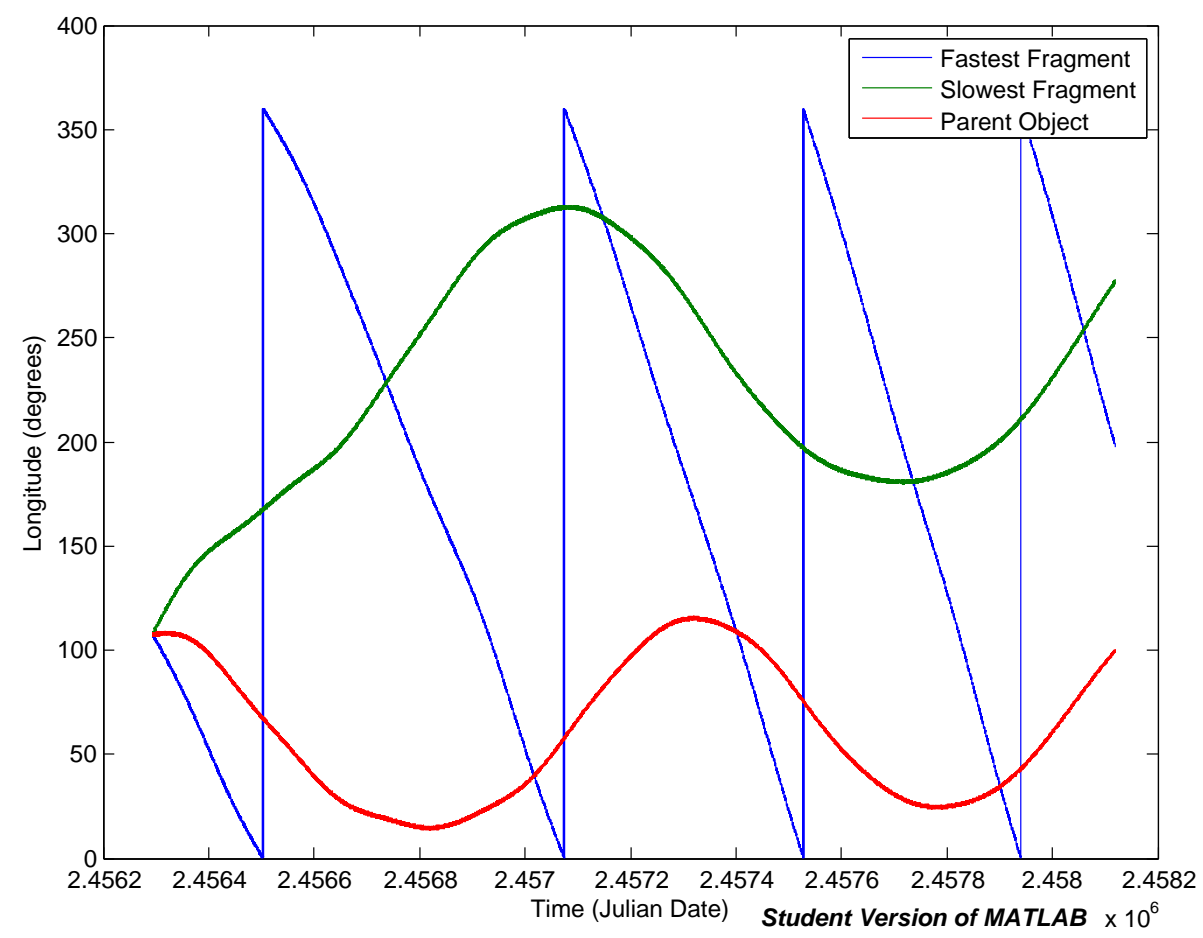

Figure 6.10: The longitudinal history of the collision's slowest and fastest fragments is shown. The slowest fragment shows trapped behavior, while the fastest object does not.

From this simulation, it can be said that the fastest fragment shows evidence against the idea of geopotential wells, particularly the West well, behaving like a "debris sink."

For both collisions, the $\Delta \Delta$ range throughout the object's orbit was observed. Figures 6.11 and 6.12 show the first 10,000 data points for the East and West well collision cases. It is important to note that McKnight defined the $\Delta \Delta$ as the relative value when the satellite is first decommissioned [1]. It was not clear if the value held after the object had become trapped.

From above, McKnight's $\Delta \Delta$ does not hold for these cases. Although some of the fragments show trapped behavior, the $\Delta \Delta$ is not less than $40 \mathrm{~km}$, which was 


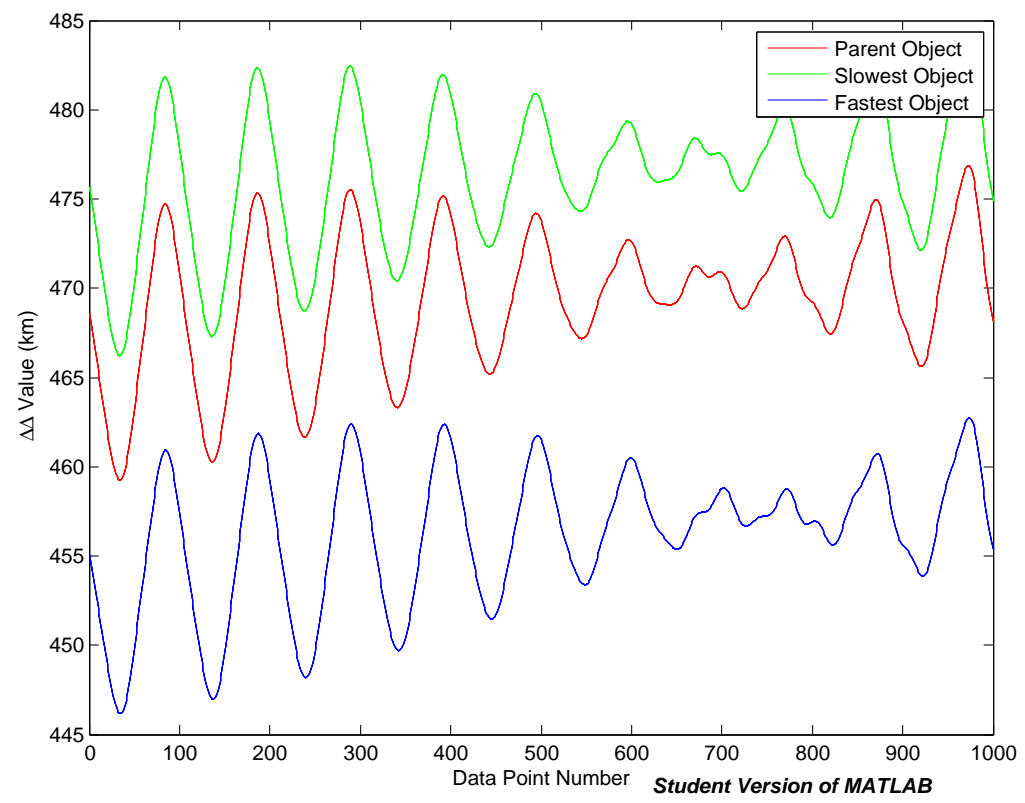

Figure 6.11: The $\Delta \Delta$ values of the East collision fragments are shown.

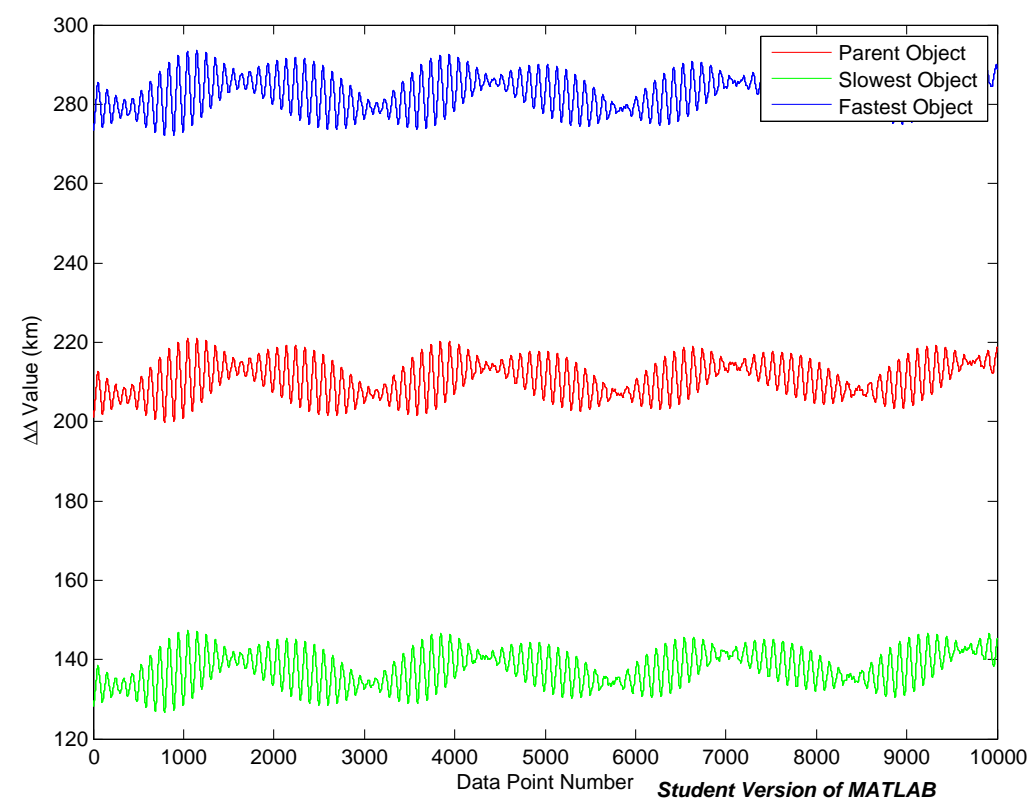

Figure 6.12: The $\Delta \Delta$ values of the West collision fragments are shown.

given as a rule a thumb. This however does not consider the longitude bin that these fragments were in initally. According to McKnight, the $\Delta \Delta$ value can be larger if 
the object is closer to a well. In this case, these objects began in the East and West wells, which could explain the higher $\Delta \Delta$ range.

\section{Concluding Remarks on Collisions}

It is thought that the differences in the collision simulations between the Eastern and Western geopotential well are due to CDPROP. CDPROP captures the Eastern and Western well objects better than the East-West well objects, despite a slight upward drift that can be seen. This upward drift must also be investigated. If fragments are shown to be trapped in the East-West wells after the collision, CDPROP will not simulate this behavior correctly, while in Figure 6.10, the West well collision, the slowest fragment does show trapping. Differences in these simulations can also be due to collision location. 


\section{Chapter 7}

\section{Conclusion and Future Work}

A Fortran 90 orbit propagator, CDPROP, or Collision Debris Propagator, was successfully developed that can be used for studying GEO disposal orbits and geopotential wells. Through verification and validation, it was determined that CDPROP could successfully predict the longitudinal history of GEO drifters and objects trapped in the the Eastern and Western geopotential well. There was also a slight upward longitudinal drift as previously mentioned which should also be investigated. It did not, however, correctly depict objects trapped between the East and West geopotential wells. It is thought to be due to the accuracy of the orbit propagation method (Cowell's method) or gravity model. This is mainly because of the accuracy of Cowell's method for longer propagations and the sensitivity of the wells to gravity model chosen. Further work into this issue may be needed. Implementing another propagation method such as Variation of Parameters that works for nearly circular orbits could possibly lower the ending residuals. Also, to increase the accuracy, study the geometric concerns with respect to solar radiation pressure (SRP) could yield a different result with the changing effect of SRP. This would be a significant effort as knowing all the geometry and material properties of an object is exceptionally difficult and not realistic at this time. Another area of improvement for computational efficiency 
is switching from MATLAB to IDL,Interactive Data Language, to increase speed for visualization.

Three test cases of collisions were also simulated using CDPROP to examine it's utility for collisions and a low velocity breakup model. The graveyard collision, after 1 year, confirmed that debris does not immediately enter into the GEO ring. The East well showed the fragments exhibiting East-West trapped behavior; however, CDPROP does not objects that are trapped between the two wells well. The West well showed the slowest fragment stayed within the West well; however, the fastest fragment showed drifting behavior, signifying it left the West well after the collision. Future work, after looking into why CDPROP does not do well in catching East-West trapped objects, is to look at how the probabilities of collision change throughout the geopotential well region and if areas of higher probability are more susceptible to losing the "debris sink" behavior. 


\section{Bibliography}

[1] D. S. McKnight and F. R. Di Pentino, "New insights on the orbital debris collision hazard at geo," International Astronautical Congress, 2011.

[2] A. C. Clarke, "Extra-terrestrial relays," Wireless World, October 1945.

[3] N. J. S. Center, "Nasa std 8719.14, nasa technical standard process for limiting orbital debris," Office of Safety and Mission Assurance, August 2007.

[4] I. S. D. C. Committee, "Iadc space debris mitigation guidelines," INTERAGENCY SPACE DEBRIS COORDINATION COMMITTEE, September 2007.

[5] J. C. V. der Ha, "Long-term evolution of near-geostationary orbits," Journal of Guidance and Control, vol. 9, 1986.

[6] N. L. Johnson, "A new look at the geo and near-geo regimes: Operations, disposals, and debris," vol. 9.

[7] N. O. D. Office, "Orbital debris optical measurements," Aug. 2009.

[8] D. J. Kessler and B. G. Cour-Palais, "Collision frequency of artificial satellites: The creation of a debris belt," Journal of Geophysical Research, 1978.

[9] H. Alfven and G. Arrhenius, "Evolution of the solar system," NASA Special Publication. SP-345, 1976. 
[10] N. L. Johnson, P. H. Krisko, J. C. Liou, and P. D. Anz-Meador, "Nasa's new breakup model of evolve 4.0," Advances in Space Research, vol. 28, 2011.

[11] T. Hanada, "Developing a low-velocity collision model based on the nasa standard breakup model," International Journal of Space Debris, vol. 2, 2000.

[12] D. A. Vallado, "Fundamentals of astrodynamics and applications," 2007. 